\title{
CONNECTED CAR NETWORKING
}

by

TENG YANG

Submitted in partial fulfillment of the requirements

For the degree of Master of Science

Department of Electrical Engineering and Computer Science

CASE WESTERN RESERVE UNIVERSITY

January, 2019 


\title{
Connected Car Networking
}

\section{Case Western Reserve University \\ Case School of Graduate Studies}

We hereby approve the thesis ${ }^{1}$ of

\author{
TENG YANG \\ for the degree of \\ Master of Science
}

\section{Dr. Christos Papachristou}

Committee Committee Chair, Adviser, Adviser

December 11, 2018

Department of Electrical Engineering and Computer Science

\section{Dr. Chris Fietkiewicz}

Committee Member

December 11, 2018

Department of Electrical Engineering and Computer Science

\section{Dr. An Wang}

Committee Member

December 11, 2018

Department of Electrical Engineering and Computer Science

\footnotetext{
${ }^{1}$ We certify that written approval has been obtained for any proprietary material contained therein.
} 
Dedicated to my parents, grandparents and my advisor Dr. Christos Papachristou 


\section{Table of Contents}

List of Tables $\quad$ vi

List of Figures $\quad$ vii

Acknowledgements $\quad$ ix

Abstract $\quad x$

Chapter 1. Introduction 1

Chapter 2. Background 4

$\begin{array}{ll}\text { Chapter 3. Approach } & 7\end{array}$

$\begin{array}{ll}\text { Definitions } & 7\end{array}$

$\begin{array}{ll}\text { Path planning } & 8\end{array}$

$\begin{array}{ll}\text { Traversal time } & 14\end{array}$

$\begin{array}{ll}\text { Traffic prediction } & 19\end{array}$

$\begin{array}{lll}\text { Chapter } 4 . & \text { Scalability } & 23\end{array}$

$\begin{array}{ll}\text { Background } & 23\end{array}$

Mapping and ports $\quad 24$

Main street and secondary street $\quad 29$

$\begin{array}{lll}\text { Chapter 5. } & \text { Results } & 32\end{array}$

Path Planning Results $\quad 32$

$\begin{array}{ll}\text { Time Estimation } & 39\end{array}$

Chapter 6. Conclusions 43 
Chapter 7. Suggested Future Research

$\begin{array}{ll}\text { Path planning } & 45\end{array}$

$\begin{array}{ll}\text { Traffic flow } & 46\end{array}$

$\begin{array}{lll}\text { Appendix A. Simulator guide } & 47\end{array}$

Appendix B. Scalability implementation guide 48

$\begin{array}{lll}\text { Appendix. } & \text { Complete References } & 53\end{array}$ 


\section{List of Tables}

3.1 Parameters of different five segments.

Car information and calculated space mean speed.

5.1 The accumulative traversal time based on path planning algorithm.

5.2 Path generating time by one main map and two local maps.

5.3 Path generating time by one main map and four local maps(max time). 


\section{List of Figures}

3.1 Traffic prediction and path planning assistance to drivers.

3.2 Initial traffic flow information.

3.3 Pre-estimation of traffic flow speeds.

3.4 Inital regular traffic example.

3.5 Traffic slow down example.

3.6 Accident example.

3.7 Five segments between Point A to Point R.

3.8 Path planning based on real-ltime traffic flow.

3.9 Three traffic snapshots based on timeline.

3.10 An example of current car changes planned path based on unpredicted blockage.

3.11 The reaction time and travel distance of each car.

4.1 An example of the main map, which can be divided into several local maps.

4.2 Local map high level graph diagram.

4.3 A graph diagram converted from the previous raw map. The start point is marked as blue point $\epsilon$ and the destination is marked as red point $\delta$. 
4.6 Different entrances (red dot) of main street (red line).

5.1 Pre-processing flow diagram.

5.2 Three simulation snapshots of preferred path based on accumulative traversal time.

5.3 Cleveland suburban test raw map.

5.4 Initialized graph and traffic.

G.5 Generated path (blue path) based on the provided map and traffic.

5.7 Another generated optimal path based on updated traffic.

5.6 An input box from the simulator for updating segment traversal time or traffic flow.

5.8 An example of traffic blockage prediction.

5.9 An example of randomly generated graph nodes of a local map.

5.10 Path generation time by one main map (red line) and two local maps (green line). 


\section{Acknowledgements}

First of all, I would like to thanks my family: my parents, grandparents and other family members for their unconditional and endless support throughout my life.

In addition to my family, I would like to express my gratitude and appreciation to my advisor Dr.Christos Papachristou for his continuous and generous guidance and help. My sincere thanks also go to Dr.Francis Wolf, who also provided important and patient support for this thesis. This thesis would not have happened without the help from them.

Last but not least, I would like to express my thanks to my thesis committee: Dr.Chris Fietkiewicz and Dr.An Wang for reviewing my thesis and providing suggestions. 


\title{
Abstract \\ Connected Car Networking
}

\author{
Abstract \\ by
}

TENG YANG

This thesis provides an efficient transportation method using connected car technology. Based on shared information communicated among vehicles, the method can provide smart navigation by two separated path planning and traffic prediction algorithms. The path planning algorithm generates the fastest path while the vehicle is moving to the destination, and the prediction algorithm dynamically updates the navigation cost of each street segment from intersection to intersection. In addition, we also discuss the parallelization of our algorithm by partitioning the large-scale metropolitan area maps into local sub-maps and addressing the scalability problem. This optimization achieves the reduction of path generation time. 


\section{Introduction}

Connected car vehicle network technology refers to a public dynamic communication system that considers communication and interaction from Vehicle to Vehicle $(\mathrm{V} 2 \mathrm{~V})^{1}$ and Vehicle to Infrastructure (V2I) ${ }^{23}$. In the connected car networking, lots of information will be shared, which includes the speed of nearby cars, the distance among nearby cars, the distance among nearby cars and the location of nearby cars. Recently, many cities have added the "time-to-green" feature for real-time traffic light information (TLI) and selected auto manufacturers have added this in some of their car models ${ }^{4}$. By this kind of connection, we can realize real-time information sharing and process this information in sub-second speeds for path planning, traffic predictive services, based on different requirements ${ }^{5}$. Furthermore, intelligent transportation solutions based on connected car technology will improve traffic, reduce accidents and reduce the energy carbon footprint.

The main motivation of this method is the need to provide an intelligent transportation solution based on connected cars. There is a need for a system of new navigation algorithms, which can assist drivers finding near optimal routing in real-time within seconds. Current car navigation methods are not based on connected cars technology. 
Current GPS-based or satellite-based applications have map limitations which are notup-to-date street maps such that do not include new streets, detours, road quality and construction lanes. Also, there is slow reporting of real-time events such as traffic signals, car braking and accidents. Real-time navigation planning should be able to handle single point destinations or multi-point destinations such as the nearest parking lot that is not full.

In this thesis, we will focus on suburban traffic problem using connected car technology. Suburban traffic in large metropolitan areas can develop particular patterns, which is different from highway patterns and downtown patterns. Suburban traffic pattern has normally traffic speed limit of $25 \mathrm{mph}$ or $35 \mathrm{mph}$ and possible accident which may block or slow down the traffic flow. We assume we have a suburban environment and we have the availability of city maps, which includes streets distances, intersections, and traffic light information (TLI). Our approach converts the city map into a graph, where the segments of the graph are road segments from intersection to intersection. The graph is directed and follows the direction of traffic. This approach is not based on GPS but could be used for enhancement. If there is a blockage in the traffic near some intersection, this method can estimate how this blockage would propagate deaccelerating of the car speed and decide if the current vehicle should wait for the obstruction or change to a new route. This method is based on the Dijkstra algorithm ${ }^{6}$ for path planning and the travel time of each street segment can be updated by current traffic information.

The objective of our work is to develop a software system to assist drivers in optimal routing in real traffic time less than a second. The navigation planning focus' is on suburban rush hour traffic in large metropolitan areas. The approach can handle blockage 
information ahead of time and can take advantage of this to re-route by using anticipatory lookahead of the mitigation effects and predictive navigation path planning. 


\section{Background}

Traditional car navigation methods are not based on connected cars. Current GPSbased or satellite-based application have several properties.

- They are location aware

- They are based on street map

- They cannot provide lookahead, anticipation or prediction, for example, cannot handle accidents in the resolution of seconds

Sharing necessary information from cars to cars could be a huge step for smart traffic planning. In this thesis, we exploit the emerging connected car technology, based on intelligent transport system standards, such as the IEEE DSRC protocol (Dedicated Short Range Communications) and some insights from the U.S. Department of Transportation's Connected Vehicle Program.

We developed a system of new navigation algorithms for assisting drivers to find near optimal routing in real-time. This intelligent transportation solution based on connected cars can

- Improve traffic and reduce accidents

- Reduce energy carbon footprint

- Provide better traffic analysis 
This system is specific for suburban path planning based on real - time traffic information provided by connected car technology since pathing planning that involves highway has other different traffic pattern.

We assume future vehicles will be provided with equipment to achieve car-to-car communications. Every car in our network has such connectivity, which is not necessarily based on WiFi protocol ${ }^{78}$ or 4 G LTE cellular ${ }^{4}$. The IEEE DSRC protocol (Dedicated Short Range Communications) $5.9 \mathrm{GHz}^{9}$ is being proposed for the emerging connected car technologies.

In addition to it, $\mathrm{V} 2 \mathrm{~V}$ communication has range of 300 meters (984 feet) and the frequency of up time 10 times per seconds according to NHTAS (National Highway Traffic Safety Administration) using DSRC. All of these protocols are collectively part of the Intelligent Transport Systems Standards 101112 .

Our proposed path planning method of connected car technology is also based on the extracted information from the real map. We converted the raw map to a diagram of the directed graph. The data schema of the graph consists of two different parts, vertices the represent the intersections and edges that represent segments of roads.

The data structure of vertices needs to contain the following:

- Primary vertices or secondary vertices (this corresponds to primary and secondary streets)

- Connectivity of vertices.

The data structure of edges needs to contain following:

- Road name

- Road length

- The number of lanes 
- Speed limitations

- Car information including speed, position and direction

- One way or two way

Some static information such as speed limit and segment length can be prepared in advance before we start the proposed path planning approach. Other dynamic information such as real-time car speed will be transmitted in real-time with connected car technology. In this case, our approach can use dynamic traversal cost calculated by real-time traffic speed to generate the optimal path.

In chapter 3, we proposed an approach to generate the path based on the shared information provided by connected car technology.

In chapter 4, we proposed a scalable optimization to implement our proposed approach in the relatively big map and reduce the path generating time.

In chapter 5, we implemented our proposed approach in Java GUI based on the particular traffic pattern and a real map. In addition to, we also discussed the performance of proposed optimization.

In chapter 6 and 7 , we provided the conclusion of this thesis and the future research suggestion in different areas.

Apart from Vehicle to Vehicle (V2V), and Vehicle to Infrastructure (V2I) as we discussed in this thesis, connected car technology also covers the emerging new areas like Vehicle to Pedestrian (V2P), Vehicle to Cloud (V2C), Vehicle to Network (V2N), Vehicle to Everything (V2X) and Autonomous cruise control (ACC). However, this work is independent of these areas. 


\section{Approach}

\subsection{Definitions}

In our approach, a car going to a destination can collect real-time $\mathrm{V} 2 \mathrm{~V}$ data from the front cars, the cars behind or the cars around, within a particular area in real time, while following the traffic queue. The normal mode of traffic queue means cars in the queue maintain constant speed and constant distance between cars, while there may be some blockage in the traffic flow. The data of each cars' current speed and position in street segments is transmitted in every second. Blockage includes all possible events that result in stopping or slowing down the traffic flow. Possible blocking or slowing down include accident, traffic light, utility cars, constructions, etc. Some kinds of blockage will be long-term, for example, utility vehicle (service truck). Other blockages will only cost a short time, for example, an animal crossing or school bus. Obviously traffic blockage may increase time.

There are two situations for the car queue to slow down. The first situation is the speed of the first car slows down to the speed of zero. The second situation is the first car slows down to a relatively low speed. The modified formulation of the first situation can still apply to the second case. Accidental stop or slow down of a specific car in this traffic 
flow will create traffic blockage "Domino effect", which results in a propagated halting or slow down of the traffic flow behind this car all the way along the traffic queue.

\subsection{Path planning}

Our path planning approach consists of two phases. The phases are represented by the two blocks of the flow chart in Fig 3.1, traffic prediction and path planning assistance to drivers. During possible blockage, the proposed method will provide traffic prediction and path planning assistance to all drivers, whether to wait for the obstruction or to take another route. The prediction and planning will keep on updating every second in realtime. Actually, we may start the path planning before we start the trip. This happens whenever we create or update the start point and end point.

The first phase requires the navigation plan to be initialized based on pre-information flow. Fig 3.2 shows the type of information that can be collected as static data from street maps, i.e. street segment length, speed limit, and conditional speed limit such as school zones or rush hour restrictions. In addition, the roads are characterized into main streets and secondary street. The main streets are used in the path planning as preferred driving routes over secondary streets in a suburban setting.

The map together with its traffic data can be converted into a navigational graph which consists of nodes representing street intersections, arcs representing street segments and other parameters for traffic information, such as speed limit. After initialization, there will be real-time traffic data like the car speed will change dynamically as the cars drive and receive new traffic information. 


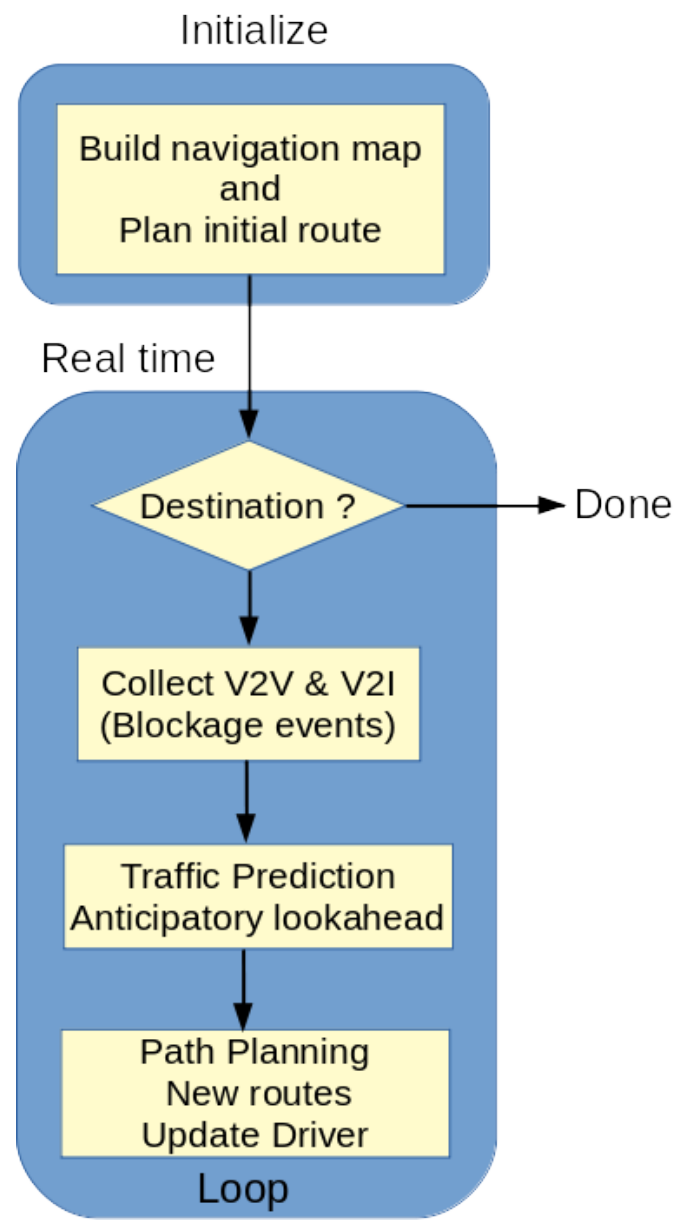

Figure 3.1. Traffic prediction and path planning assistance to drivers.

By traversal time, we mean the time required for a car to go from its current position to the end of the current street segment. Segment traversal time is the time required for a car to drive from the start intersection of a street segment to the end intersection of it.

Fig 3.3 shows a portion of a street map annotated with the initial segment traversal times. In the static traffic flow, each segment traversal time is computed by a simple velocity formula using the street segment length divided by the speed limit ${ }^{13}$. In this example of Fig 3.3, we assume a route from node A to node $\mathrm{R}$ and each segment has a speed limit of 35 miles per hour. Segments AF, FN, NQ and QR have lengths of 0.2, 0.3, 
0.3 and 0.1 miles. Thus initially segment traversal time estimates result in 20, 30, 30 and 10 seconds. This information is annotated to each segment (Fig 3.3).

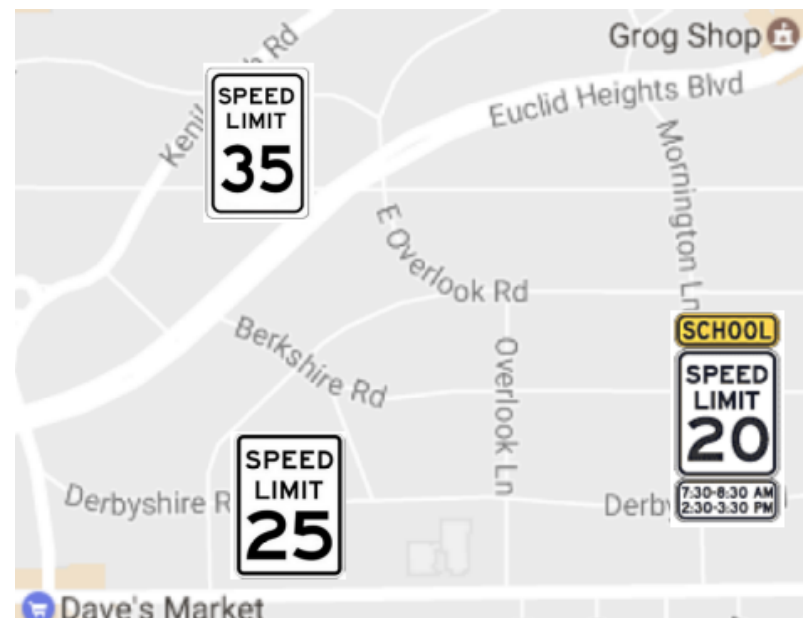

Figure 3.2. Initial traffic flow information.

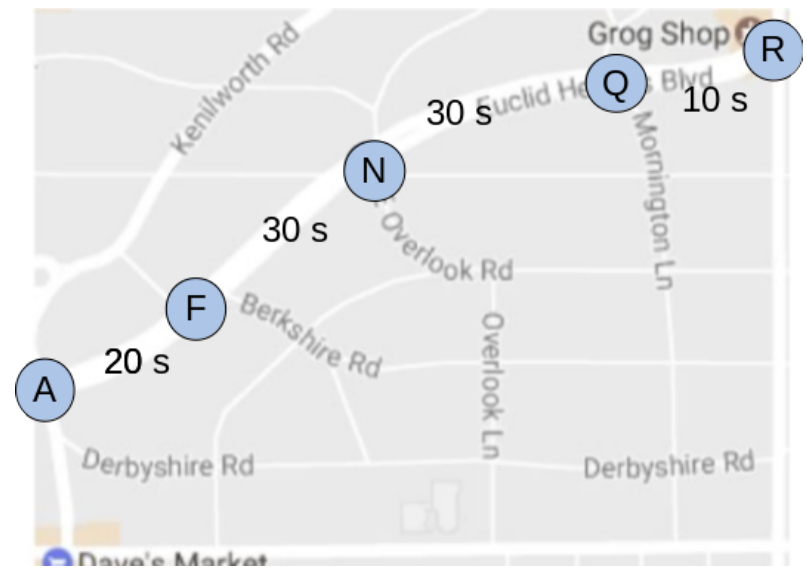

Figure 3.3. Pre-estimation of traffic flow speeds.

The second phase in Fig 3.1 begins by updating the navigational map in real-time. This includes collecting blockage events as they occur from V2V and V2I sources. As mentioned, the navigational path planning consists of two steps, planning and prediction. The navigation map is computed based on anticipatory lookahead due to blockage events such as multiple cars braking. The new traffic predictions allow our system to 
generate new possible routes. This leads to alerting the driver of any better route options until the destination has been reached.

The planning step can be conducted in every traversal. To be more specific, every time a vehicle moves to the next node (intersection), we can perform a new calculation of planning and a newly updated navigation time (from start to destination of the trip). The tentative new path may require updates.

Because of the possible selection of different paths due to real-time traffic, we implemented the 'dynamic' Dijkstra's shortest path algorithm ${ }^{6}$. In every iteration, we take the segment traversal time as the cost of the Dijkstra's shortest path algorithm to generate the 'shortest' path based on the current traffic situation.

Fig 3.4 shows how the path planning algorithm can handle the dynamically updated traversal time. Suppose node A is the start node and G is the end node. A is marked as visited. The annotated value between two adjacent nodes represents the traversal time. The green number on the top of some nodes are the current total traversal time from the current car position to this node.

All nodes contain different traversal different total traversal time from the start point to this node based on the different tentative paths. In our example of Fig 3.4, node A has two neighbors: B and F. In the first two iterations, the visited nodes are A, B, C, G, F, N. The current optimal path is $\mathrm{A} \rightarrow \mathrm{B} \rightarrow \mathrm{G}$. 


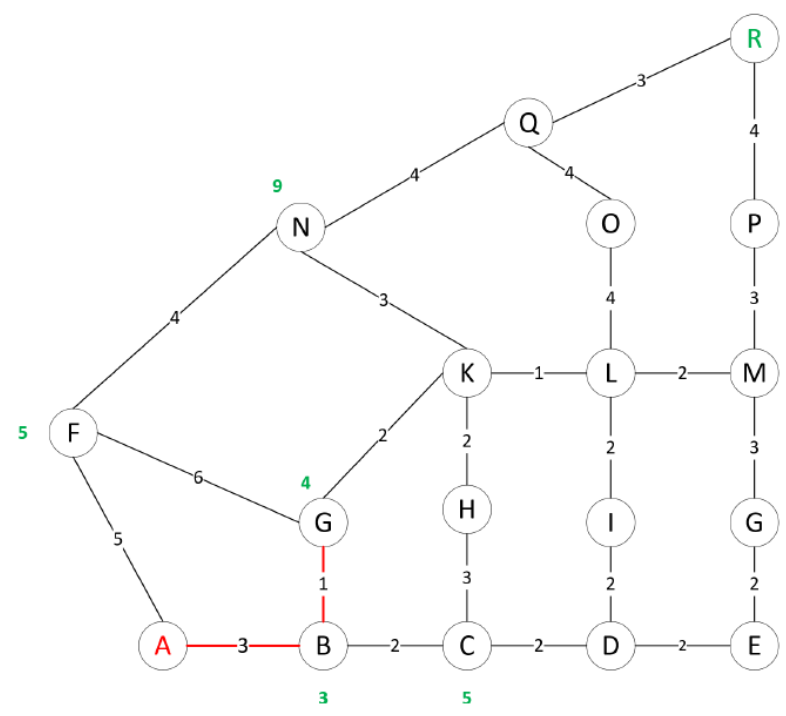

Figure 3.4. Inital regular traffic example.

Fig 3.5 shows a traffic slow down example. The segment traversal time from node $G$ to node $\mathrm{k}$ increased from 2 to 3 and the segment traversal time from node $\mathrm{C}$ to node $\mathrm{D}$ increased from 2 to 4 due to traffic slow down. This information is transmitted based on updated connected car technology. However, the current path $A \rightarrow B \rightarrow G \rightarrow K$ still maintains the shortest path. We can also notice that both the path $A \rightarrow B \rightarrow G \rightarrow K \rightarrow$ $\mathrm{N}$ and the path $\mathrm{A} \rightarrow \mathrm{F} \rightarrow \mathrm{N}$ share the same cost of 9 . If the destination is $\mathrm{N}$, we will have two paths. 


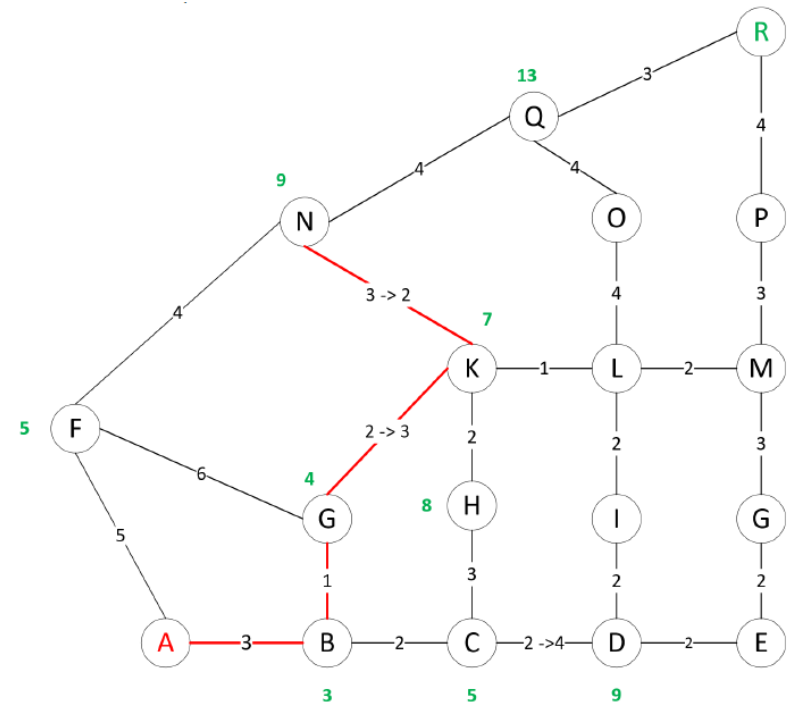

Figure 3.5. Traffic slow down example.

Fig 3.6 exhibits a blockage example. We can assume that traffic accident happened in the segment between $\mathrm{L}$ and $\mathrm{M}$ and sharply increased the traversal time from 2 to 7 . All nodes that is next to R, Q can provide lowest accumulated cost, which is 13 . Then the final cost of traveling from $A$ to $R$ is 16 and the final path is $A \rightarrow B \rightarrow G \rightarrow K \rightarrow N \rightarrow Q \rightarrow$ R.

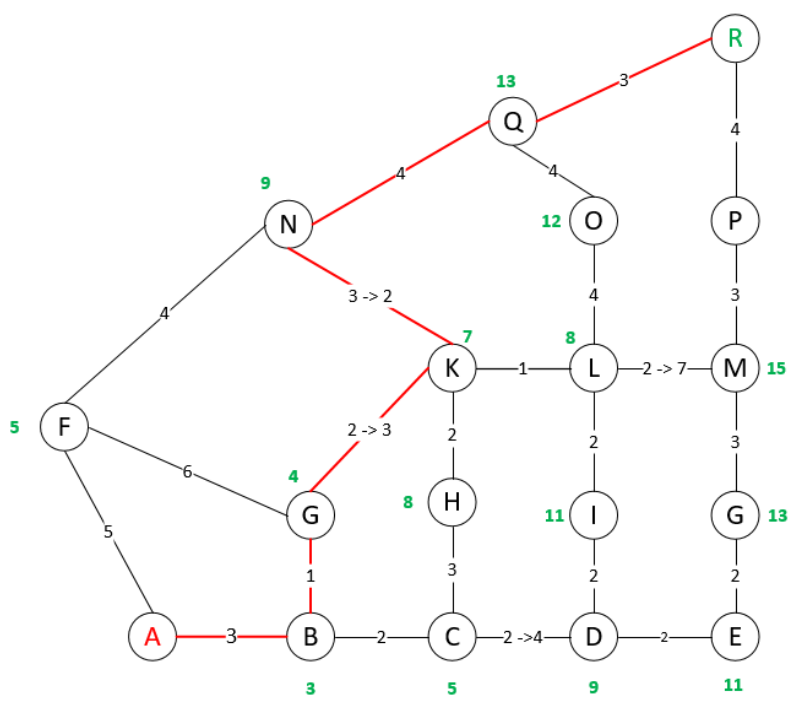

Figure 3.6. Accident example. 


\subsection{Traversal time}

Path planning without traffic is much easier. In Fig 3.7, we assume one car is driving from point A to point $\mathrm{R}$, the path from A to $\mathrm{R}$ can be divided into five segments. Table 3.1 shows the related traffic data of these five segments.

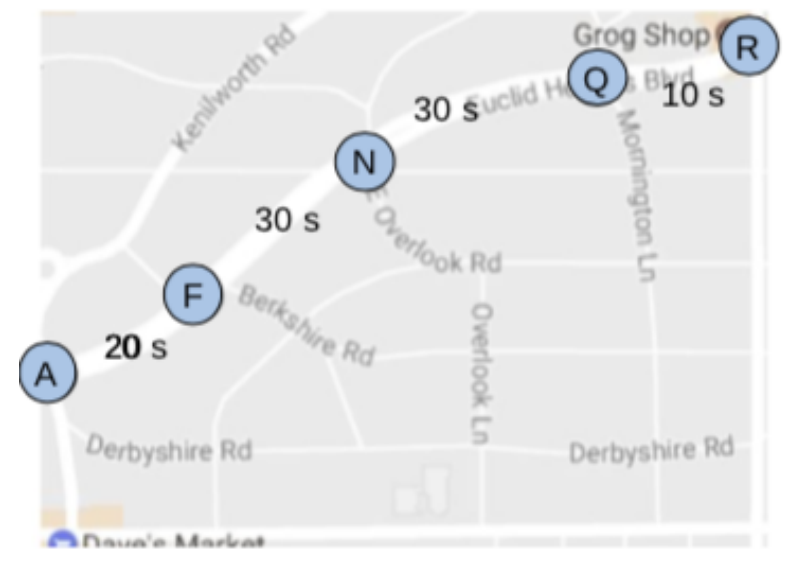

Figure 3.7. Five segments between Point A to Point R.

Segment Distance(miles) Speed Limit(miles/hour) Segment traversal Time(seconds)
A - F $\quad 0.2$
35
20
F - N $\quad 0.3$
35
30
$\mathrm{N}-\mathrm{Q} \quad 0.3$
35
30
Q - R
0.1
35
10

Table 3.1. Parameters of different five segments.

In one car driving condition, the segment traversal is static and can be given by

$$
t_{\text {segment }_{i, j}}=\frac{d_{\text {segment }_{i, j}}}{v_{\text {segment }_{i, j}}}
$$


In the formulation, $t_{\text {segment }}$ rij represents the segment traversal time from node $\mathrm{i}$ to node $\mathrm{j}, d_{\text {segment }_{i, j}}$ represents the segment length from node i to node $\mathrm{j}$ and $v_{\text {segment }} \mathrm{t}_{i, j}$ represents the speed limit from node i to node j. For example,

$$
t_{A, F}=\frac{d_{A, F}}{v_{A, F}}=\frac{0.2 m}{35 m p h}=20 s
$$

Compared with previous static traversal time, space mean speed is measured over the whole segment ${ }^{14}$ based on all cars' speed on the current segment to provide dynamic segment traversal time based on real-time traffic flow and is consider more accurate to represent the traffic flow compared to time mean speed ${ }^{14}$. Space mean speeds exhibits the harmonic mean of speeds passing a segment during a period of time. $v_{i}$ represents the real-time speed of the $i_{t h}$ car and $\mathrm{n}$ represents the number of car in this segment. Suppose there is a segment with $\mathrm{n}$ cars, the calculation of space mean speed $\bar{v}_{s}$ is given by

$$
\bar{v}_{s}=\frac{n}{\sum_{i=1}^{n} \frac{1}{v_{i}}}
$$

For example, given five observed velocities (60 mph, $35 \mathrm{mph}, 45 \mathrm{mph}$ ), the space mean speed $\bar{v}_{s}$ will be

$$
\bar{v}_{s}=\frac{N}{\sum_{n=1}^{N} \frac{1}{v_{n}}}=\frac{3}{\frac{1}{60}+\frac{1}{35}+\frac{1}{45}}=44.47 \mathrm{mph}
$$

Fig 3.8 provides the workflow of generating the optimal path based on real-time segment traversal time calculated by space mean speed. 


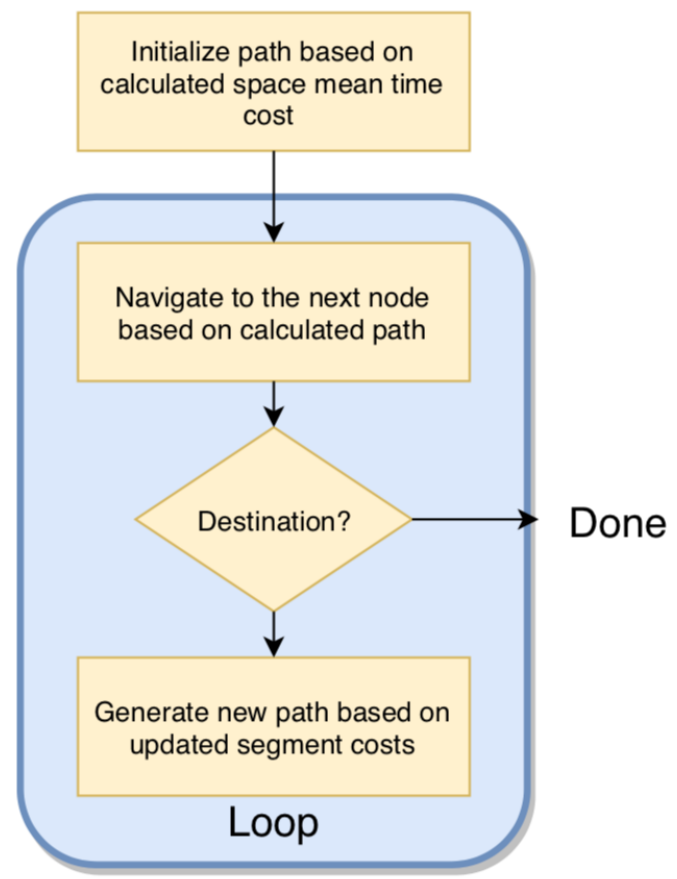

Figure 3.8. Path planning based on real-ltime traffic flow.

We provide three snapshots of traffic as three different scenario of real-time traffic update in a time-based order. Fig 3.9 shows the traffic snapshot based on timeline, each red square represents a car with different speed and destination, but in a same traffic flow towards node E. Car information in different segment is shown in Table 3.2

We can assume that one car starts from departure A to destination E at Time $t$. Based on the instant cost of each segment from A to E, we can calculated the cost from A to $\mathrm{E}$ is

$$
\operatorname{Cost}_{(A B C D E)}=23.6+34.8+35.5+13.2=107 \text { seconds }
$$

Fig 3.9 assumes this car arrives at B at time t3. Since the cost of each segment is keeping on updating, we can conduct a new calculation of shortest path algorithm in each nodes based on dynamic cost. Based on the calculated instant segment traversal cost, 


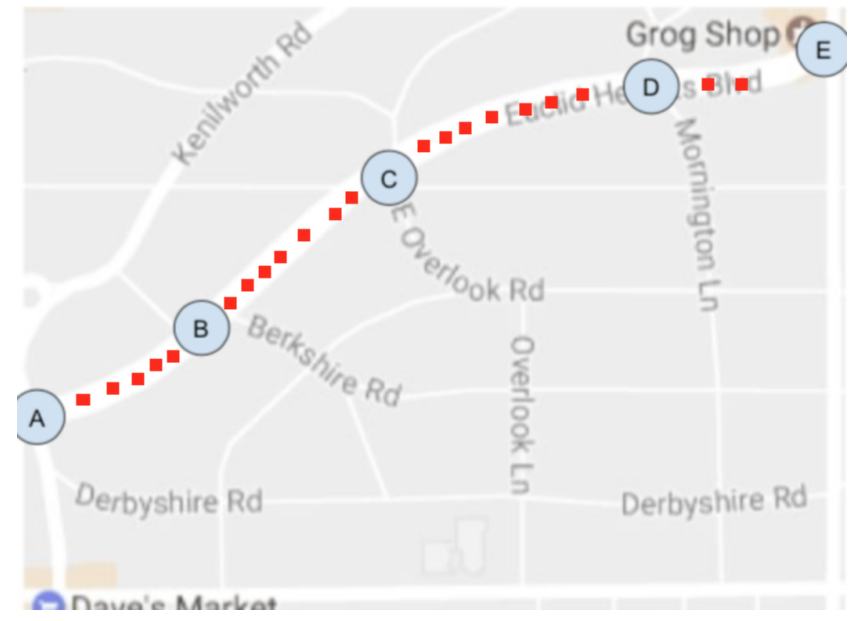

(1)

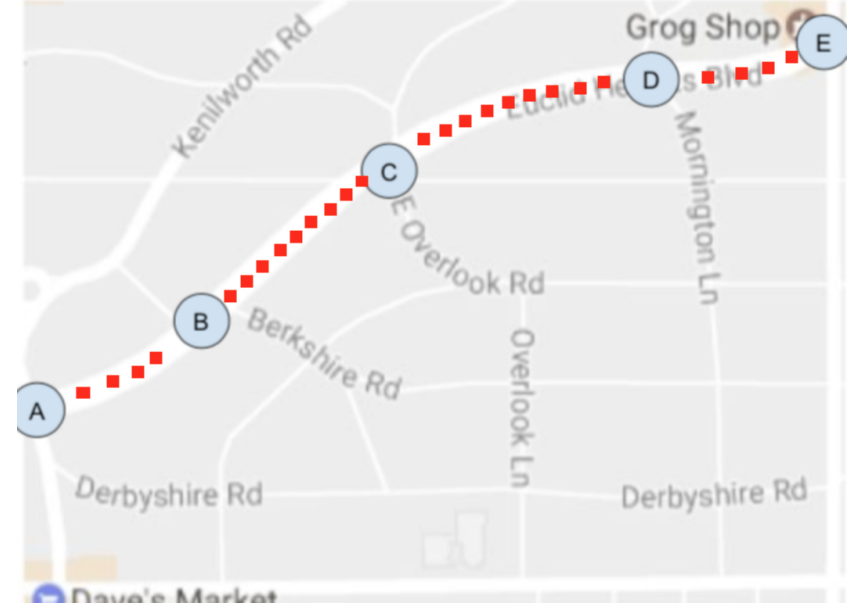

(2)

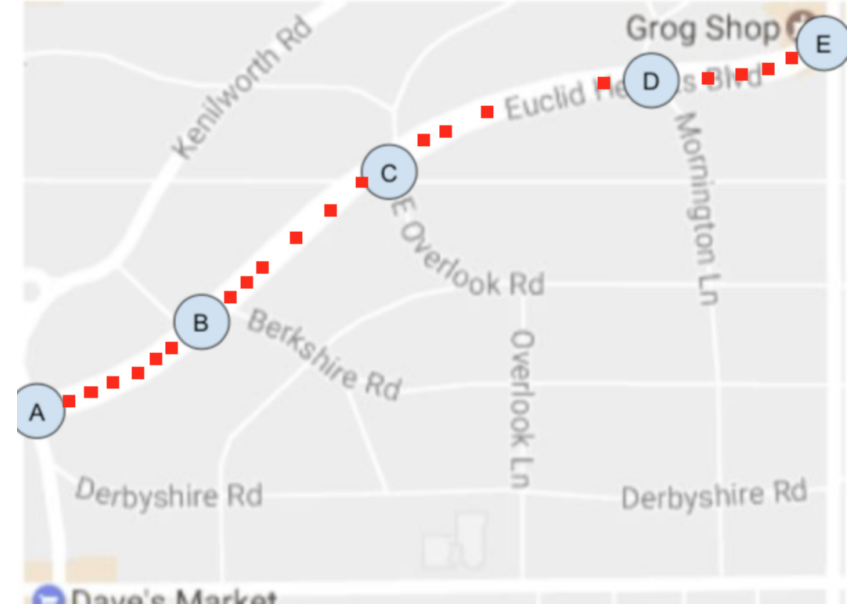

(3)

Figure 3.9. Three traffic snapshots based on timeline. 


$\begin{array}{llll}\text { Segment } & \text { Cars with Different Speed (mile/hour) } & \begin{array}{l}\text { Space Mean Speed } \\ \text { (mile/hour) }\end{array} & \text { Estimated Time (second) } \\ \text { Time t1 } & & 30.5 & 23.6 \\ \text { A - B } & 35,30,25,35,30 & 31.0 & 34.8 \\ \text { B - C } & 35,30,25,35,30,35,30 & 30.4 & 35.5 \\ \text { C - D } & 35,30,25,35,30,30,30 & 27.3 & 13.2 \\ \text { D - E } & 30,25 & & \\ \text { Time t2 } & & 30.7 & 23.5 \\ \text { A - B } & 35,30,25,35 & 30.0 & 35.9 \\ \text { B - C } & 35,30,25,35,30,35,30,30,25 & 26.7 & 40.5 \\ \text { C - D } & 35,30,25,35,30,30,30,25,15 & 19.4 & 18.6 \\ \text { D - E } & 30,25,15,15 & & \\ \text { Time t3 } & & 28.7 & 38.1 \\ \text { A - B } & 35,30,25,35,35,20 & 28.1 & 135.0 \\ \text { B - C } & 35,30,25,35,30,20 & 8 & 18.6 \\ \text { C - D } & 10,10,10,5 & 19.4 & \\ \text { D - E } & 30,25,15,15 & \end{array}$

we can notice that some slow down is happening in segment $C D$, which will increase the traversal time from B to E to

$$
\operatorname{Cost}_{(B C D E)}=38.5+135.0+18.6=192.1 \text { seconds }
$$

However this path planning method explores all the alternative paths in the map. In Fig 3.10, if segment CF has a cost of 80.0 and segment EF has a cost of 20.0, we can notice that the alternate path B - C - F - E (blue line) provides lower cost compared to path B C - D - E (blue dash line). In this case, we can conclude that the most efficient path from $\mathrm{B}$ to $\mathrm{E}$ is path $\mathrm{B}-\mathrm{C}-\mathrm{F}-\mathrm{E}$ at time $\mathrm{t} 3$.

$$
\operatorname{Cost}_{(B C F E)}=38.6+80.0+20.0=136.8 \text { seconds }
$$




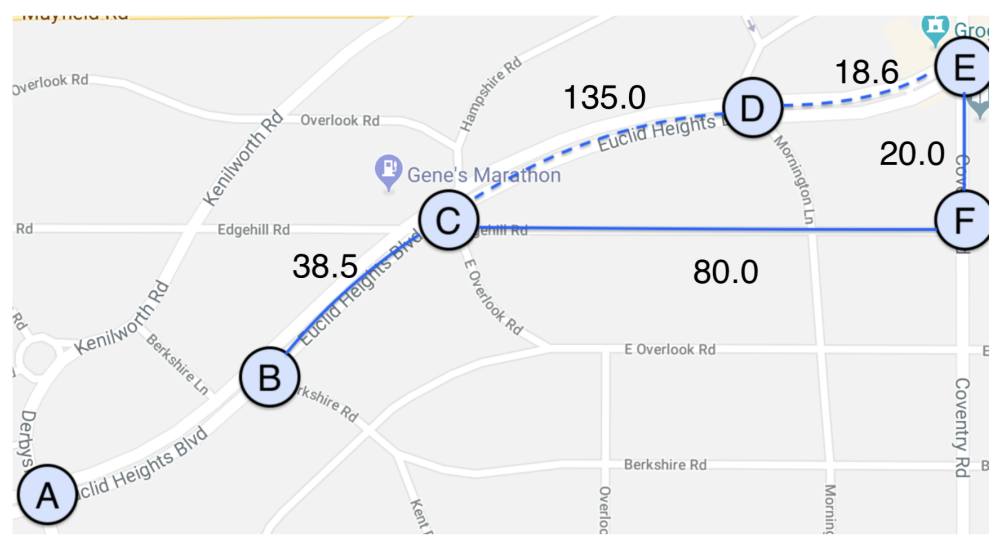

Figure 3.10. An example of current car changes planned path based on unpredicted blockage.

\subsection{Traffic prediction}

The previously proposed approach provides the path planning solution based on realtime traffic in the scope of the map. The map consists of a large set of segments. We can optimize the proposed path planning algorithm in the level of a single segment. For example, a serious car accident could result in a complete blockage of the current segment. But at the time when this accident occurs, other cars may still maintain a certain speed until they are forced to slow down to stop. It's possible to predict this blockage and avoid this route in advance.

As we proposed before, the navigation path is calculated by the connectivity between nodes and the cost. The cost represents the segment traversal time. The time can be predicted based on following considerations. The number of cars before our car is $n$. The length of each car is $d_{c}$. The initial distance in the queue between the first car and the $n$th car is $d_{\text {total }}$. The initial distance between the first car and the second car is $d_{1}$. The initial distance between the first car and the third car is $d_{2}$. The reaction time is $t_{r}$. All cars travel with the same speed of $\nu_{0}$. If the first car reduces the speed to 0 , 
all following cars will also speed down to 0 . The deceleration of the all cars is $a$. The distance the first car travels after the car start braking reaction is $S_{1}$. The distance the $n$th car travel after the first car start reaction is $S_{n}$. The distance of the car travels during the reaction time and the braking time is $s_{r}$ and $s_{b}$ respectively.

Consider a blockage affecting a queue of cars. In order to simplify the problem, we assume each driver has the same reaction time ${ }^{15}$. Suppose the first car has reaction time of $t_{r}$. At the end of $t_{r}$, the second car will see the first car's blockage(breaking) and it will start to reduce speed. The second car has reaction time of $2 t_{r}$. We can conclude that, the $n$th car will have reaction of $n \cdot t_{r}$ and, all cars travel same distance to the stopping other cars. The relationship of how each car is positioned in the queue can be formulated based on reaction time as shown in Fig 3.11.

In this situation of car queue compression: The travel distance of the first car after the accident is $d_{r 1}=s_{r}+s_{b}$. The distance of the second car after the accident is $d_{r 1}=$ $2 s_{r}+s_{b}$. The distance of the $n$th car after the accident is $d_{r n}=n s_{r}+s_{b}$. We notice that each car will shorten the distance between by $s_{r}$. We can conclude that

$$
\begin{gathered}
s_{1}=d_{r 1}=s_{r}+s_{b} \\
s_{n}=d_{r n}=n s_{r}+s_{b} \\
s_{n}-s_{n-1}=s_{n-1}-s_{n-2}=s_{3}-s_{2}=s_{2}-s_{1}=s_{r} \\
d_{2}=d_{1}-s_{r}
\end{gathered}
$$



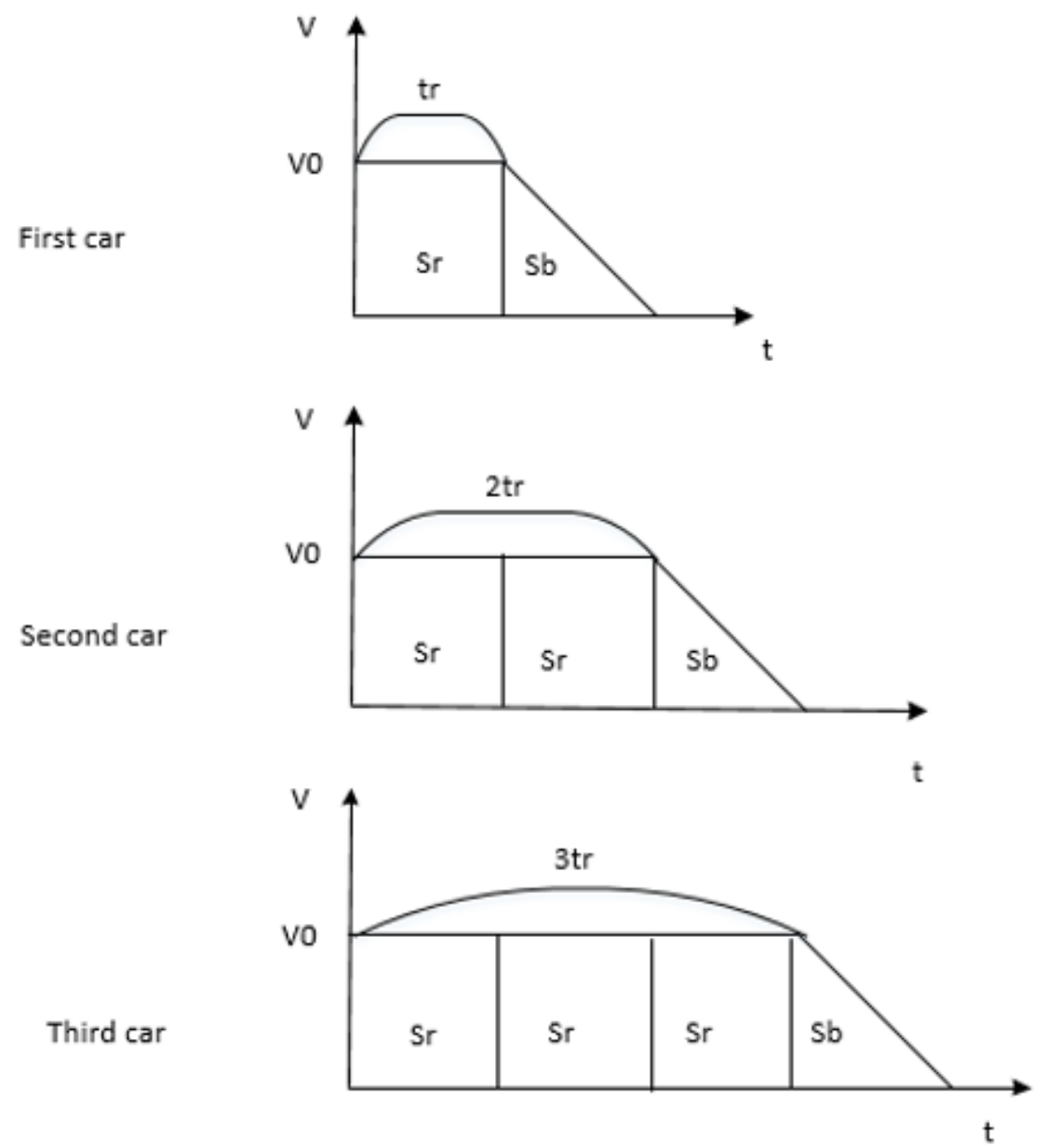

Figure 3.11. The reaction time and travel distance of each car.

In Fig 3.11, $v_{0}$ is the initial speed of the car. $t_{r}$ is the reaction time. $s_{r}$ is the distance that a car travels during the reaction time. $s_{b}$ is the distance that a car travels during the braking time. Based on these conditions, we can calculate several parameters: The initial distance between our car and the first car is

$$
d_{\text {total }}=(n-1) \cdot d_{1}+(n-1) \cdot d_{c}
$$

The brake time is

$$
t_{b}=v_{0} / a
$$


The breaking and reaction distance of the first car is

$$
S_{1}=S_{r}+S_{b}=v_{1} \cdot t_{r}+\left(a \cdot t_{b} \cdot t_{b}\right) / a
$$

The total distance for $n$th car to travel when the first car started reducing the speed from $v_{0}$ to 0 is

$$
\begin{aligned}
& S_{n}=d_{\text {total }}+S_{1}-(n-1) \cdot d_{2}-(n-1) \cdot d_{c} \\
& =n \cdot\left(d_{1}-d_{2}\right)+S_{1}=(n-1) \cdot S_{r}+S_{1} \\
& =n \cdot S_{r}+S_{b}
\end{aligned}
$$

In this case, we notice that using our basic approach, if we forecast that the first car is trying to reduce the speed, we need to reduce the speed of our car to the same speed in the distance of $S_{n}$ and

$$
t_{\text {prediction }}=\frac{n \cdot s_{r}}{\nu_{0}}+\frac{s_{b}}{\frac{\nu_{0}}{2}}=n \cdot t_{r}+t_{b}
$$

So, we can predict that in the time of $t_{\text {prediction, }}$ our car will reach the final speed. The time or prediction can be used to prevent the car from getting stuck into traffic jam caused by nearby accident.

Assume the current car position is Node K and an accident happens between Node L and Node M, which will reduce the speed of traffic flow to zero based on Fig 3.6. In this case, based on the number of cars between Node K and Node L, we can calculate the prediction time, which stands for when the traffic speed between $\mathrm{K}$ and $\mathrm{L}$ also reduce down to zero. If the prediction time is relatively small, the current car may change its tentative path based on a updated calculation of shortest path algorithm. 


\section{Scalability}

\subsection{Background}

Current path planning method is designed to handle path planning problems in the suburban area based on dynamically updated traversal time. Implementing the path planning algorithm in the real situations requires solving the scalability issue. This requires to scale our method to handle larger city area maps or the entire city.

In our proposed scalability solution, a single path planning model requires to choose the optimal path of a map calculated by a single process. In reality, the traditional path planning algorithm in a big map may require much time to be processed by a single path planning model. However, we can divide the big city map into several small local maps and calculate the paths separately. In the proposed parallel path planning algorithm, 1) we will calculate the cost of departure to the connected intersections between local maps, 2) take these connected intersections as new departure nodes and 3) compute the new cost of next local map in the same way. Real situation path planning using the previously proposed method in the compound big map can use this parallel path planning approach to increase path generation processing speed. 


\subsection{Mapping and ports}

The whole map used for path planning is identified as the main map. The main map can be partitioned into different contiguous local maps and processed by separate path planning models. Local maps inside the main map can use a path planning model which includes departures, destinations and segment traversal time. The perimeter of the local map is identified as the side. Side contains ports between adjacent local maps and separates the local maps from each other. The shape of the local map does not necessarily have to be a quadrangle.

This main map dividing method will be parallelized to reduce processing time and provide a faster solution for path planning in the bigger map. In the local map, we still take each part of the street segment between intersection and intersection as a segment. Each segment still uses segment traversal time as traversal cost for path processing.

For example, in Fig 4.1, we want to traverse in a bigger map from one place in Shaker Heights (marked as blue point $\epsilon$ ) to Kelvin Smith Library (marked as red point $\delta$ ). We can partition our main map into seven local maps, notice we have several preferred paths inside each local map. In order to get the best path from the departure in local map A to the destination in local map G, we will generate several alternatives based on path planning algorithm applied to different maps and select the best path from them.

The connection between local maps can be represented by a higher level graph. This kind of higher level graph diagram consists of local maps as verticles and connectivity between adjacent local maps as edges. The cost (weight) of these edges can be represented by average total traversal time from a group of randomly picked departures or destinations from two adjacent local maps using standard deviation. These costs between local map are used to evaluate which permutation of locals map may provide the 
optimal path and prevent generating the route towards the wrong direction. In the example of Fig 4.2, the graph exhibits fours ways to traversal from local map A to local map B. They are $A-C-D-F-G, A-C-D-E-G, A-B-D-E-G, A-B-D-F-G$ separately. We choose the ports that are collinear from the departure to the destination.

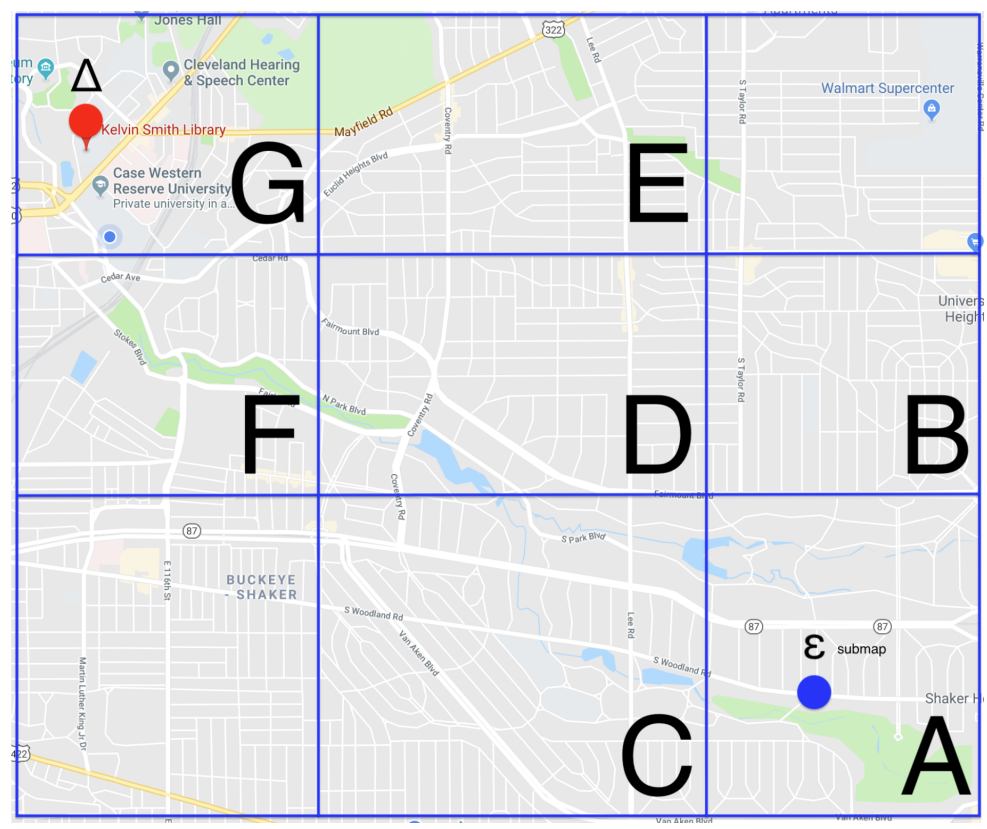

Figure 4.1. An example of the main map, which can be divided into several local maps.

Fig 4.3 represents a graph diagram converted from the previous main map. The crossing boundary between two adjacent maps above the crossing point is a port, which provides communication of processed paths including cost among maps. An example of ports derived from the previous map is shown in Fig. 4.4. Assume that local map A has three ports connected to B and two ports that are connected to C. We annotate these ports respectively as $P_{A B 1}, P_{A B 2}, P_{A B 3}$ and $P_{A C 1}$ and $P_{A C 2}$. In addition to it, the 


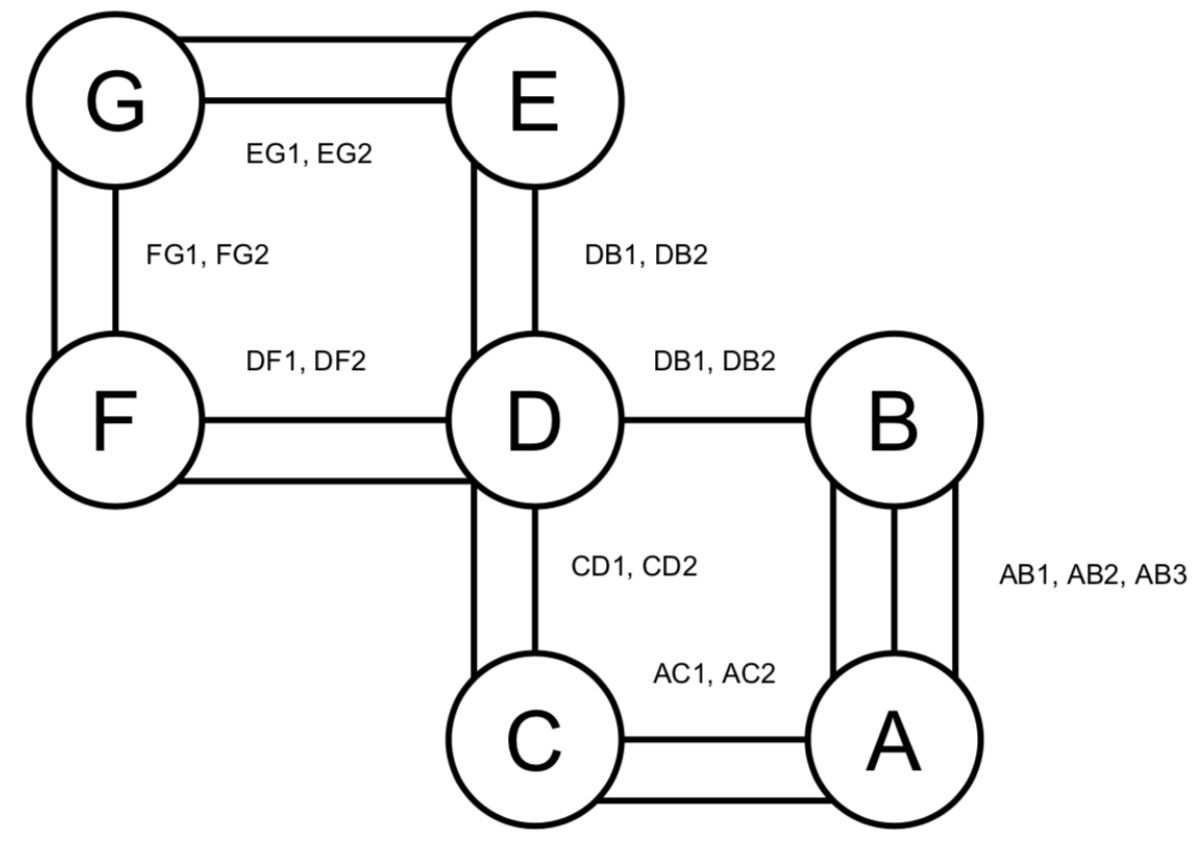

Figure 4.2. Local map high level graph diagram.

start point in the main map is annotated as $\epsilon$, and the endpoint is annotated as $\Delta$. The cost from the start point to the destination is annotated as $C_{\epsilon, \Delta}$. Path planning among maps requires several steps.

First, several paths will be generated from the start point to the common ports of the local map and its adjacent local maps. In our example, these local maps are map B and map C. These ports are served as local destinations with calculated path planning cost. We can calculate the cost of the following paths, $C(\epsilon, A B 1), C(\epsilon, A B 2), C(\epsilon, A B 3)$, $C(\epsilon, A C 1), C(\epsilon, A C 2)$.

Second, based on the calculated initial cost, we can obtain the best path from the departure to $\mathrm{AB} 1, \mathrm{AB} 2, \mathrm{AB} 3$, and $\mathrm{AC} 1$ and $\mathrm{AC} 2$. Based on these values, we can calculate the best path from these ports to other ports that are adjacent to other maps, which are marked as $C D 1, C D 2, B D 1$ and $B D 2$ regarding local maps $\mathrm{C}$ and $\mathrm{D}$ respectively in our 


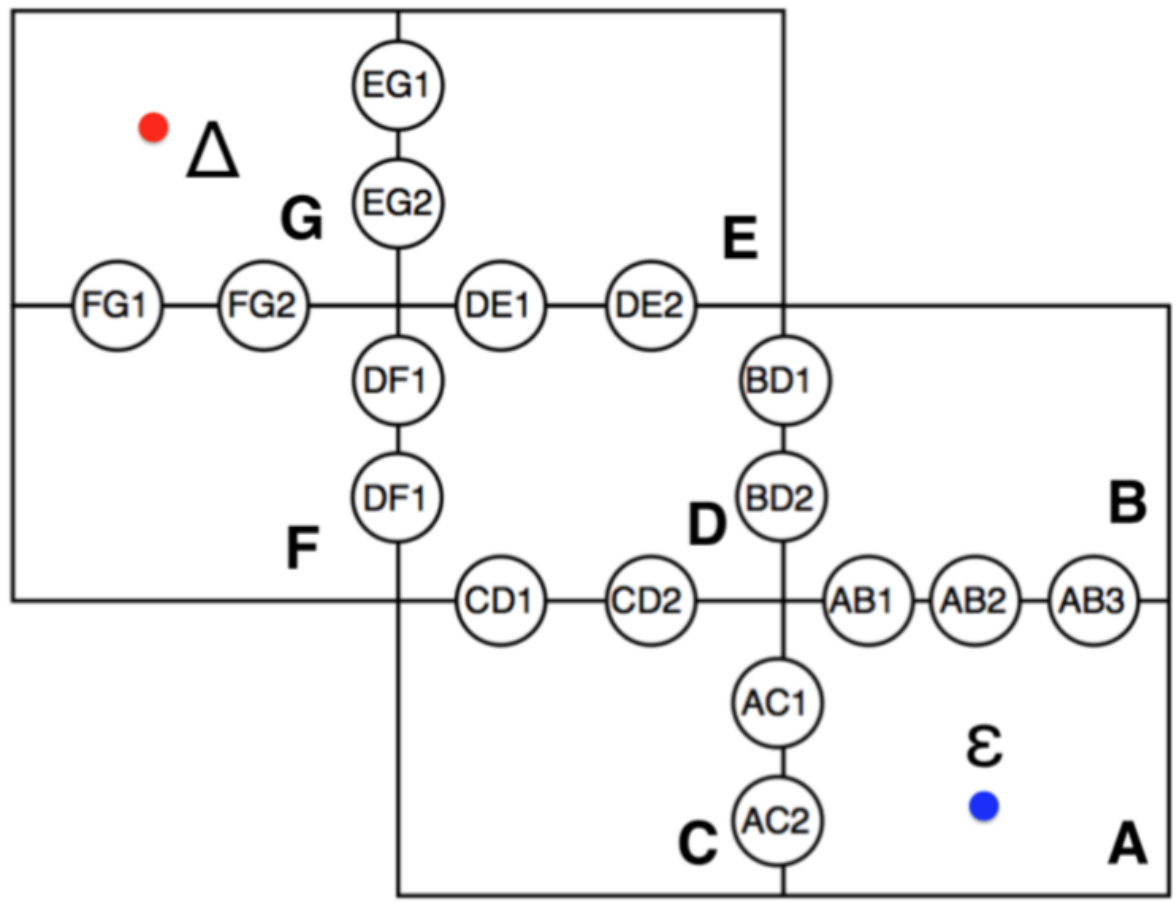

Figure 4.3. A graph diagram converted from the previous raw map. The start point is marked as blue point $\epsilon$ and the destination is marked as red point $\delta$.

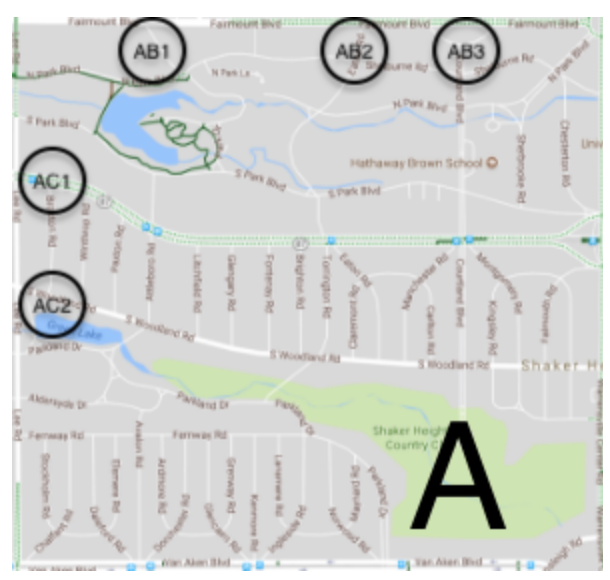

(a) Raw local map A.

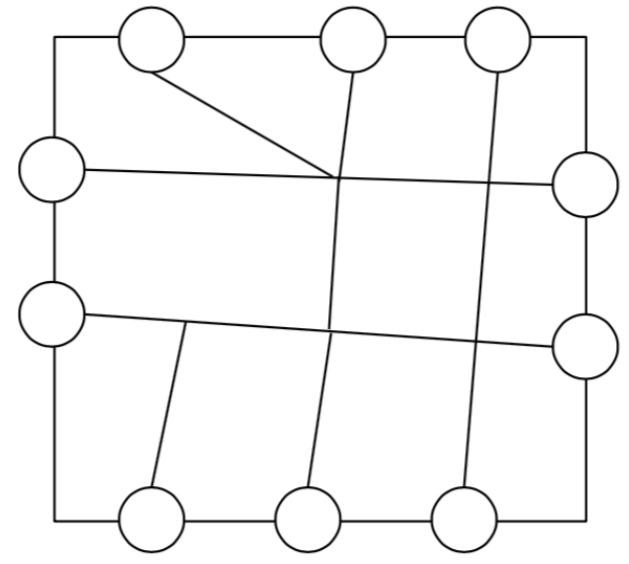

(b) Local map A graph diagram.

Figure 4.4. An example of map A, which is a part of example main map.

example. 
Thus we have the following costs from start to ports

$$
\begin{aligned}
& C(\epsilon, C D 1)=\min (C(\epsilon, A C 1)+C(A C 1, C D 1) ; C(\epsilon, A C 2)+C(A C 2, C D 1)) \\
& C(\epsilon, C D 2)=\min (C(\epsilon, A C 1)+C(A C 1, C D 2) ; C(\epsilon, A C 2)+C(A C 2, C D 2))
\end{aligned}
$$

$$
\begin{array}{r}
C(\epsilon, B D 1)=\min (C(\epsilon, A B 1)+C(A B 1, B D 1) ; \\
C(\epsilon, A B 2)+C(A B 2, B D 1) ; \\
C(\epsilon, A B 3)+C(A B 3, B D 1)) \\
C(\epsilon, B D 1)=\min (C(\epsilon, A B 1)+C(A B 1, B D 2) ; \\
C(\epsilon, A B 2)+C(A B 2, B D 2) ; \\
C(\epsilon, A B 3)+C(A B 3, B D 2))
\end{array}
$$

Based on the same idea, $P_{C D 1}, P_{C D 2}, P_{B D 1}$, and $P_{B D 2}$ will serve as the new departures for generating paths in map D towards the new destination of $P_{D F 1}, P_{D F 2}, P_{D E 1}$, and $P_{D E 2}$. In the final map G, new departures ports $P_{E G 1}, P_{E G 2}, P_{F G 1}$ and $P_{E G 2}$ will be used to calculate the best path towards the final decision in the map G. In the end, the cost of the smallest cost path from the departure to destination will be 


$$
\begin{array}{r}
C(\epsilon, \Delta)=\min (C(\epsilon, E G 1)+C(E G 1, \Delta) ; \\
C(\epsilon, E G 2)+C(E G 2, \Delta) ; \\
C(\epsilon, F G 1)+C(F G 1, \Delta) ; \\
C(\epsilon, F G 2)+C(F G 2, \Delta))
\end{array}
$$

The time required to generate the optimal path can be reduced because the cost computing in each local map can be processed asynchronously. The steps of calculating the cost among ports are independent of each other. For example, the cost from AC1 to CD2 is not based on other maps but will be used in calculating the optimal path to CD1 and CD2. Summing up all the minimal cost between ports will provide the best path from the departure to the destination on the main map. The result of this example is shown in Fig 4.5.

\subsection{Main street and secondary street}

The street segments that we use for path planning consist of the main streets and the secondary streets based on street properties and drivers preferences. The main street is a street that a) contains at least 2 lanes in each direction, b) has speed limit is $35 \mathrm{mph}$ or higher. The secondary street is the street that a) has no more than two one lanes in each direction, b) has speed limit is $25 \mathrm{mp}$ or lower.

An example of main street is shown in Fig 4.6. In our path planning approach, the main street has higher priority when a car travels in a relatively big map due to the higher 


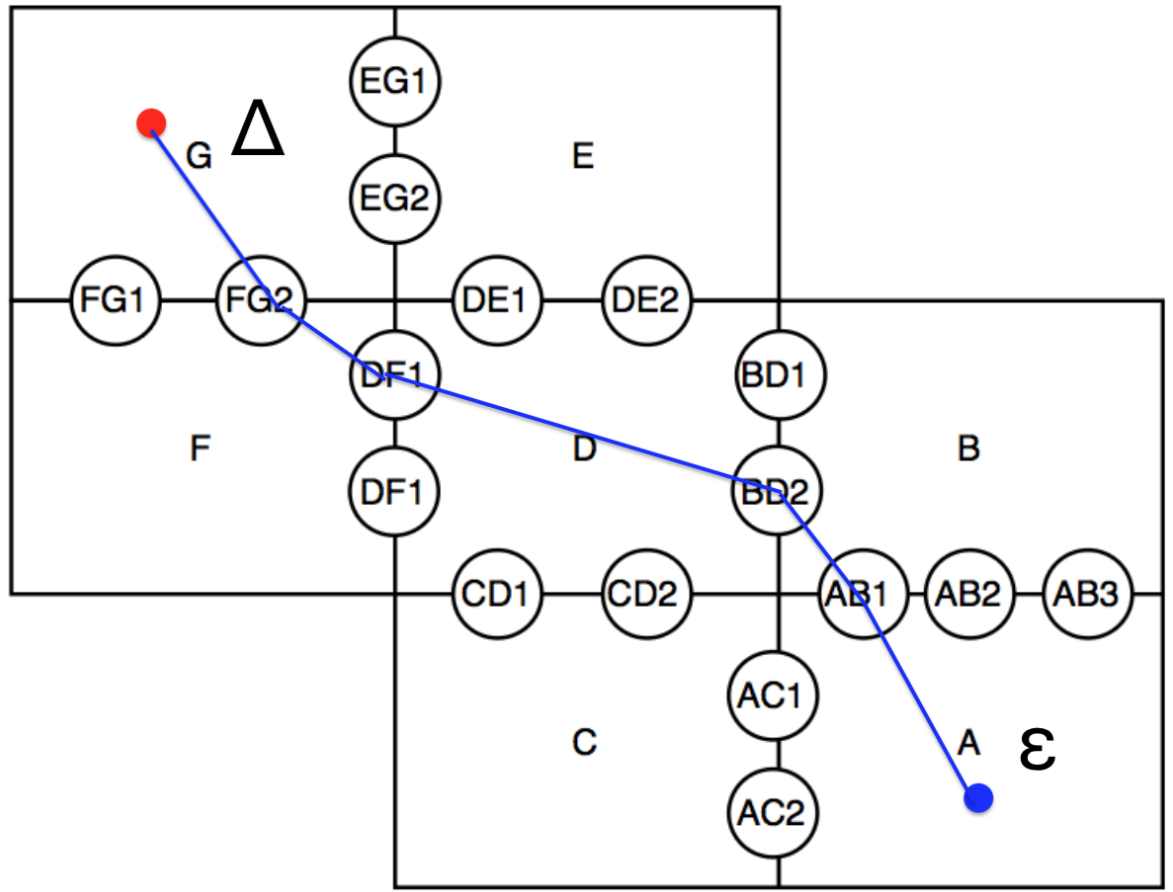

Figure 4.5. A possible generated optimal path.

speed limit, driver habit, etc. It's not necessary to consider the secondary street when we conduct path planning in big maps. The main street exclusive path planning can reduce the path generating time. However, the departure as well the destination is normally on the secondary streets when we start path planning at the beginning. For example, in Fig 4.6, if the departure address is 2649 Berkshire Rd, we will have several options to navigate to different main street entrances (marked as the red dot). Based on the segment traversal time of these secondary streets, we can still perform total traversal time calculation to these main entrances using the path planning algorithm previously discussed (chapter 3). Because the algorithm calculates the cost of all divergent path, this path generation only needs to be performed once. The entrances with the shortest cost will be used to provide as main local street departure. 


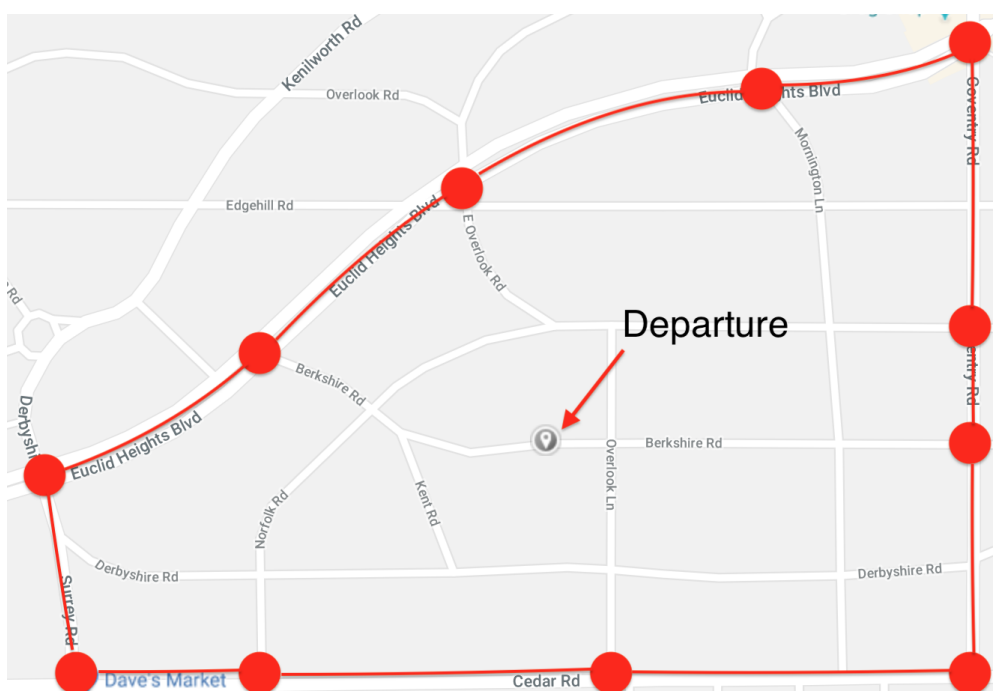

Figure 4.6. Different entrances (red dot) of main street (red line). 


\section{Results}

\subsection{Path Planning Results}

Based on the path planning and prediction algorithms, we can conclude that the proposed will generate an optimal navigation path with lowest navigation cost each time when the vehicle starts to traverse to a next intersection.

We implemented our connected car algorithms based on the proposed path planning method which includes planning, prediction and simulation and developed our system software in Java using the Java GUI Library to illustrate the different path planning solutions based on different traffic patterns.

Our pre-processing work includes the extraction of the city map and conversion into a graph diagrams with the annotated main/secondary streets, intersections, speed limit, and other relevant parameters. Besides, we initiated traffic patterns based on our experienced evidence from observing the traffic situations in our city area. Fig. 5.1 shows that the flow of our software system that initializes traffic patterns and pre-defined graphs as input, then combine it with the proposed path planning and traffic prediction method to start a path planning. 


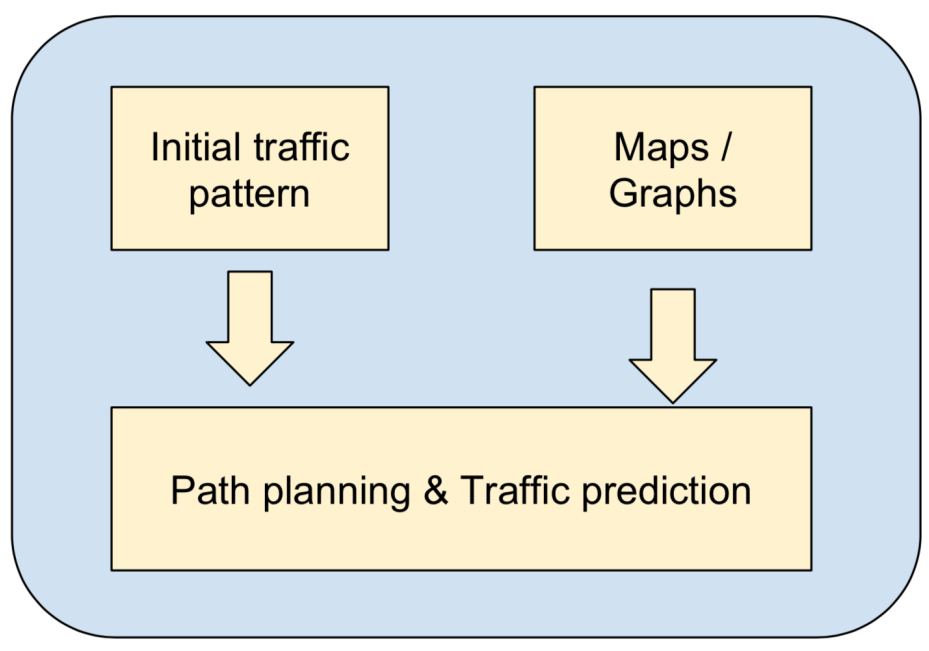

Figure 5.1. Pre-processing flow diagram.

Our results are shown in Fig. 5.2 in reference to Table 5.1, where we perform the previously proposed path planning algorithm discussed in Chapter 3 in different traffic called traffic 1, traffic 2 and traffic 3. Table 5.1 exhibits the accumulated traversal cost of each node based on this approach. In Fig. 5.2, Node A is the departure and Node R is the destination. Each street segment has segment traversal time calculated by the realtime traffic speed and road length. The segment traversal cost is marked as black inside the street segment. The blue number next to each node represents its accumulative traversal time. The preferred path is shown as the red line. In traffic 1 , we can see there are two optimal paths in this area. Both the path $\mathrm{A} \rightarrow \mathrm{F} \rightarrow \mathrm{N} \rightarrow \mathrm{Q} \rightarrow \mathrm{R}$ and the path $\mathrm{A} \rightarrow \mathrm{B} \rightarrow \mathrm{C} \rightarrow \mathrm{D} \rightarrow \mathrm{E} \rightarrow \mathrm{G} \rightarrow \mathrm{M} \rightarrow \mathrm{P} \rightarrow \mathrm{R}$ can perform the fastest traversal, which will take 16 normalized time units (a time units mean the time required to traverse from one intersection to another intersection). The traversal path may be different if there are traffic blockages or accidents. 


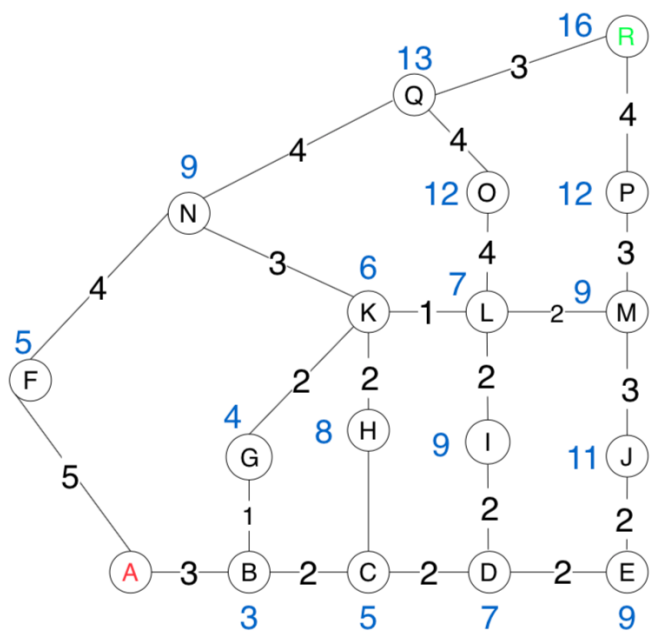

(a) Traffic 1

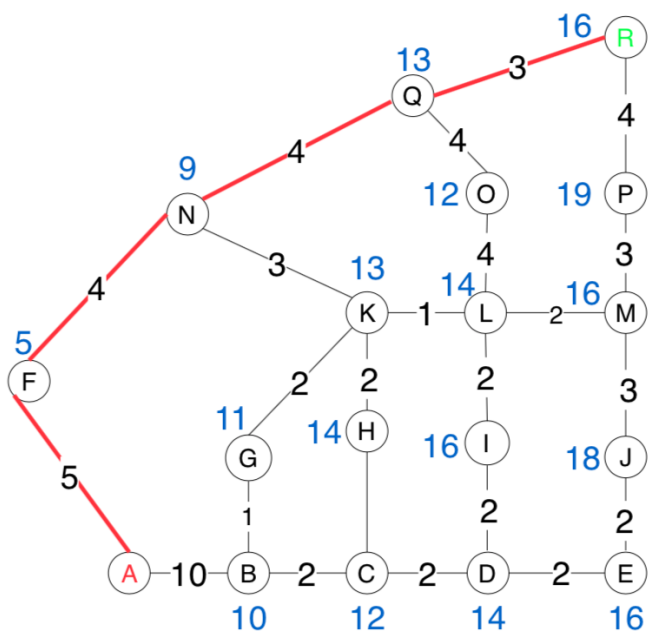

(b) Traffic 2

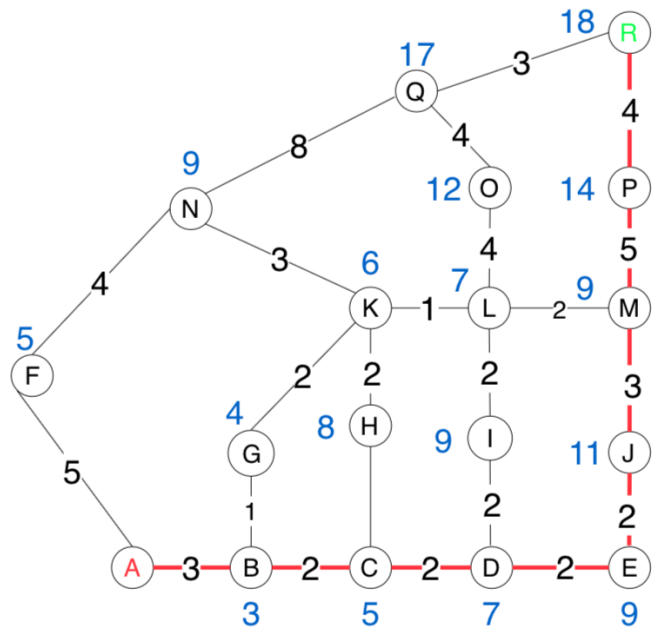

(c) Traffic 3

Figure 5.2. Three simulation snapshots of preferred path based on accumulative traversal time. 
Node Traffic 1 Traffic 2 Traffic 3

$\begin{array}{llll}\mathrm{A} & 0 & 0 & 0 \\ \mathrm{~B} & 3 & 10 & 3 \\ \mathrm{C} & 5 & 12 & 5 \\ \mathrm{D} & 7 & 14 & 7 \\ \mathrm{E} & 9 & 16 & 9 \\ \mathrm{~F} & 5 & 5 & 5 \\ \mathrm{G} & 4 & 11 & 4 \\ \mathrm{H} & 8 & 14 & 8 \\ \mathrm{I} & 9 & 16 & 9 \\ \mathrm{~J} & 11 & 18 & 11 \\ \mathrm{~K} & 6 & 13 & 6 \\ \mathrm{~L} & 7 & 14 & 7 \\ \mathrm{M} & 9 & 16 & 9 \\ \mathrm{~N} & 9 & 9 & 9 \\ \mathrm{O} & 12 & 12 & 12 \\ \mathrm{P} & 12 & 19 & 14 \\ \mathrm{Q} & 13 & 13 & 17 \\ \mathrm{R} & 16 & 16 & 18\end{array}$

Table 5.1. The accumulative traversal time based on path planning algorithm.

For example, in traffic 2, we assume that there is a blockage between Node A and Node B resulting in a delay of 7 time units. The navigation cost will be updated from 3 to 10 . The complete navigation path cost of $\mathrm{A} \rightarrow \mathrm{B} \rightarrow \mathrm{C} \rightarrow \mathrm{D} \rightarrow \mathrm{E} \rightarrow \mathrm{G} \rightarrow \mathrm{M} \rightarrow \mathrm{P} \rightarrow \mathrm{R}$ will be 23 time units. But the path $\mathrm{A} \rightarrow \mathrm{F} \rightarrow \mathrm{N} \rightarrow \mathrm{Q} \rightarrow \mathrm{R}$ will still be the fastest route with the minimum cost of 16 .

For traffic 3, if there is an accident between Node Q and R, which will increase the navigation cost of this path from 3 to 5 , the path $A \rightarrow B \rightarrow C \rightarrow D \rightarrow E \rightarrow G \rightarrow M \rightarrow P \rightarrow$ $\mathrm{R}$ will be the only one optimal way for navigation, but the final traversal time wouldn't change. If there are two accidents in path from $\mathrm{N}$ to $\mathrm{Q}$ (4 to 8 ) and $\mathrm{M}$ to $\mathrm{P}$ (3 to 5), the generated path will be $\mathrm{A} \rightarrow \mathrm{B} \rightarrow \mathrm{C} \rightarrow \mathrm{D} \rightarrow \mathrm{E} \rightarrow \mathrm{G} \rightarrow \mathrm{M} \rightarrow \mathrm{P} \rightarrow \mathrm{R}$, based on increased cost from 16 to 18. 


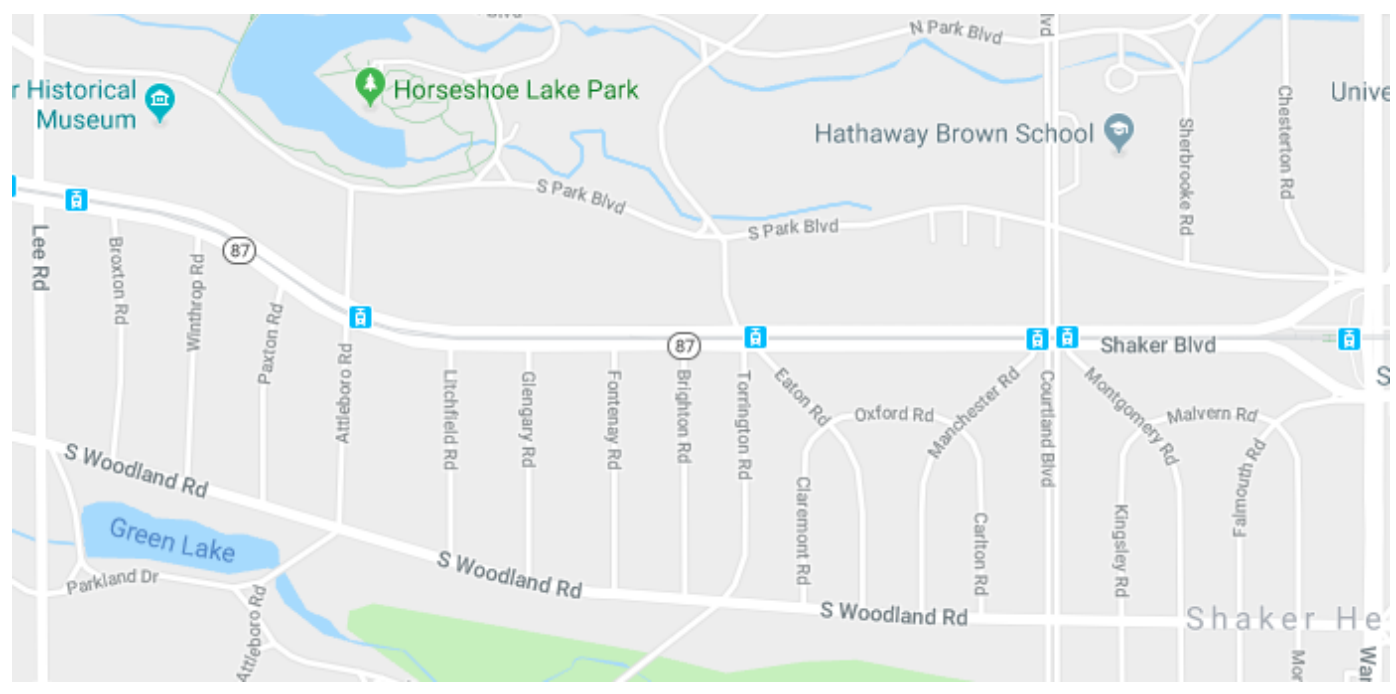

Figure 5.3. Cleveland suburban test raw map.

The proposed method has been implemented in a software simulator ${ }^{16}$ using Java GUI based on Java Swing ${ }^{17}$ and Java AWT ${ }^{18}$. Our simulator has been tested in suburban traffic of Cleveland using real raw maps. One example map fragment shown in Fig 5.3. In our simulator, each map is converted to a graph diagram with nodes, street segments and segment traversal times as shown in Fig 5.4. Each street is revealed by a black line with a black node in the middle of it representing its segment traversal time. The purple nodes are the street intersections. If we define it as departure or destination, its color will change into blue or red respectively. The blue path exhibits the generated preferred path. If we provide the departure (node 15) and destination (node 41), the simulator will provide the current optimal path based the existing traffic as shown in Fig 5.5.

The simulator reads a CSV file containing the name of the street, the connectivity of the street intersection and the segment traversal time of each street segment. We can edit the CSV input to update the structure of the map and change the traffic pattern. Besides, We can also update the segment traversal time by providing particular values or cars' speed and road length using the input box shown in 5.6. For example, we can 


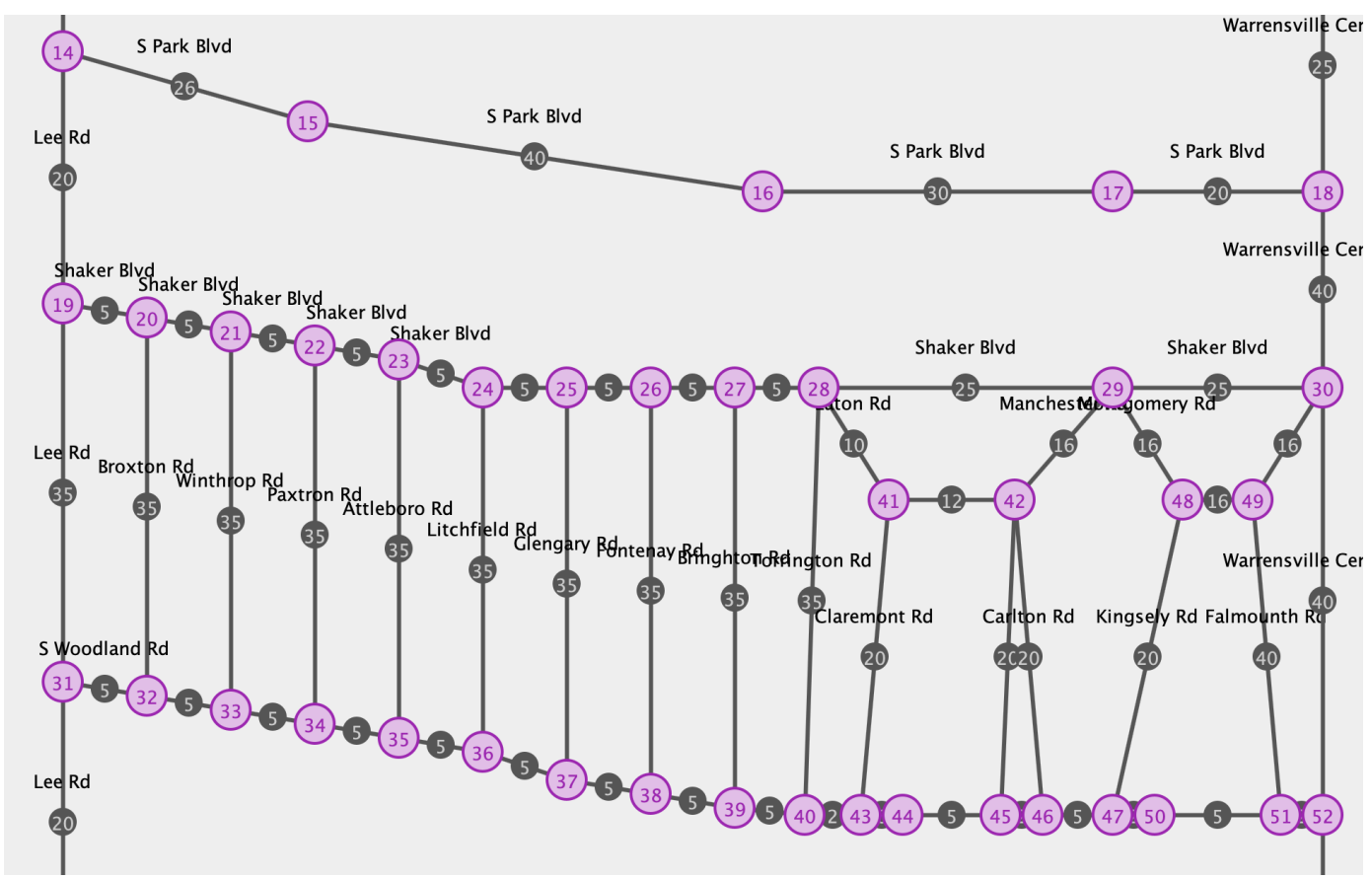

Figure 5.4. Initialized graph and traffic.

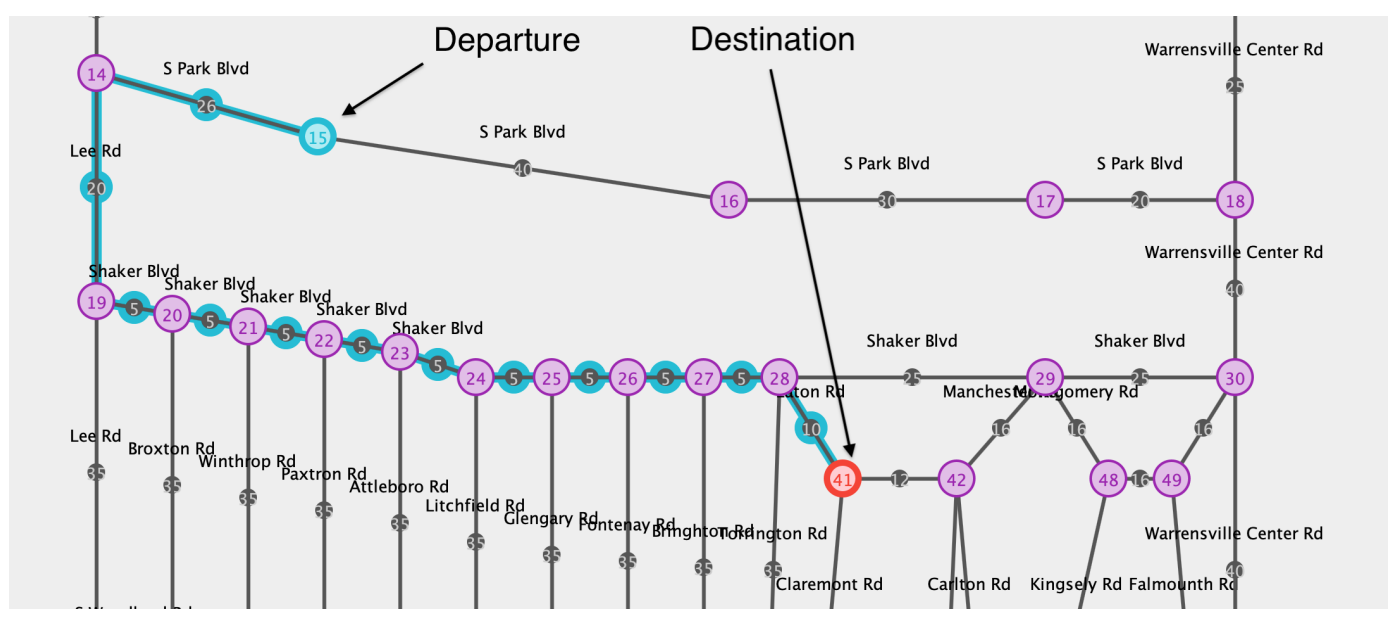

Figure 5.5. Generated path (blue path) based on the provided map and traffic.

provide the traffic parameters to alternate the traffic between node 10 and node 14 (part of Lee Rd). Assume a traffic slow-down is happening in this specific segment, i.e., three cars in this segment have slowed down to the speed of $4 \mathrm{mph}, 5 \mathrm{mph}, 6 \mathrm{mph}$, separately and the length of this specific segment is one mile. The simulator provides an alternative 


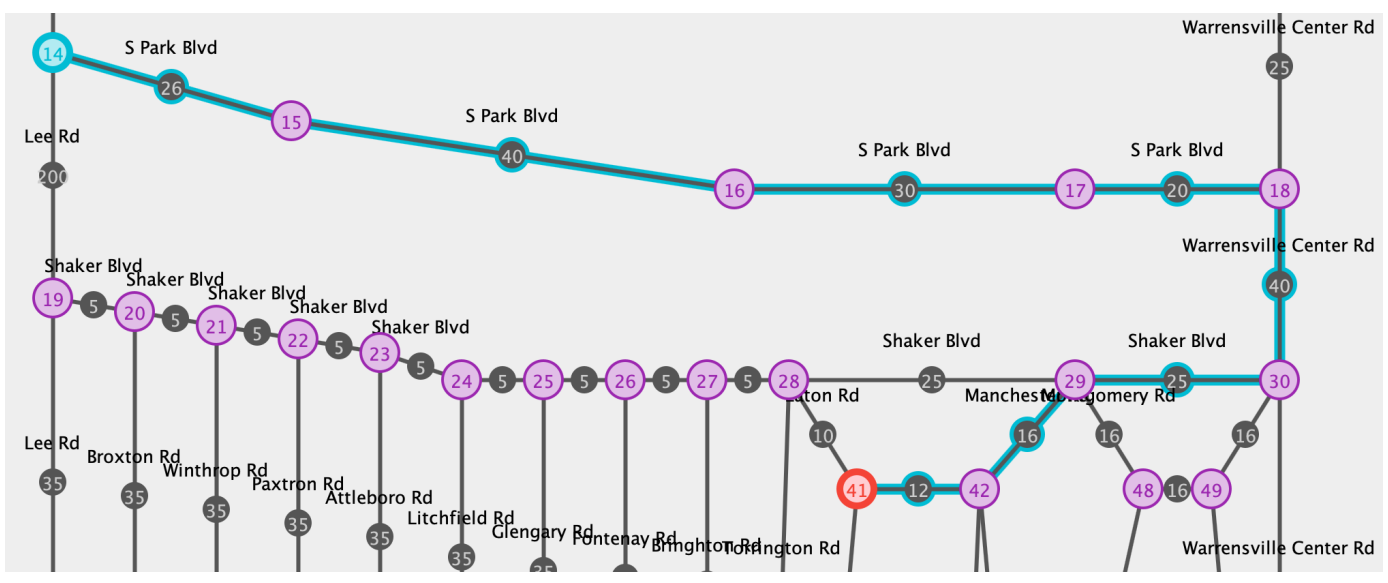

Figure 5.7. Another generated optimal path based on updated traffic.

path passing through "Warrensville center road" based on the updated traffic as show Fig 5.7.

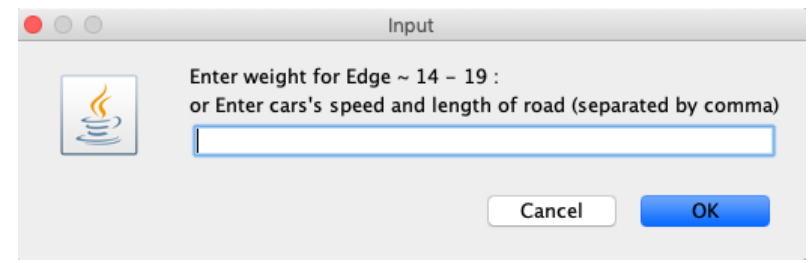

Figure 5.6. An input box from the simulator for updating segment traversal time or traffic flow.

In addition to path planning, the simulator can also provide traffic prediction based on traffic flow on each segment to avoid waiting by taking the alternative route. It also provides guidance. For example, in Fig 5.8, if a car accident happened, the simulator will predict how long the traffic queue will reach the reduced speed by using the prediction time formula provided previously (Chapter 3).

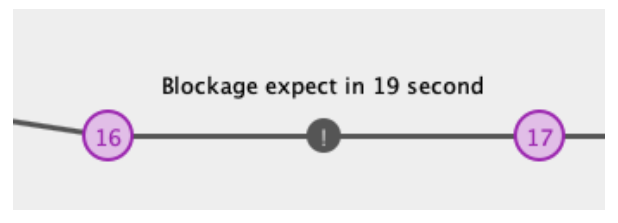

Figure 5.8. An example of traffic blockage prediction. 


\subsection{Time Estimation}

We have generated a random and static main map for testing the performance of our scalability approach for path planning. In this main map, every node in the graph has two, three or four neighborhood intersections connected by street segments. The traversal cost of each street segment between nodes was also randomly generated with segment traversal time from 0 to 100 . The main will be divided into local maps equally. For example, a four by four randomly generated local map graph nodes is shown in Fig 5.9 .

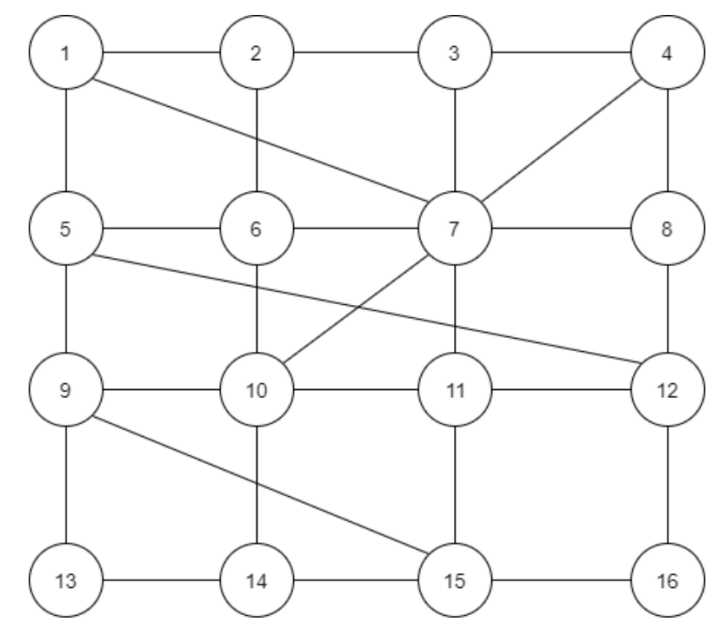

Figure 5.9. An example of randomly generated graph nodes of a local map.

Based on the generated main map, we can validate the performance of our previous scalability path planning method. As we discussed previously (Chapter 3), each path generation model is independent of each other. Several optimal paths inside the local map are generated from ports to ports first until the final optimal path is summed up from them.

We evaluated the efficiency of this method by diving the main map into two local maps and generating the best path using two path generation models and compare the 
path generation time with the time using only one map path generation model. The divided local maps are connected to each other by four ports. Each map path generation model is calculated by one process. Each process is also independent of each other during calculation. We verify the correctness of the outcome by checking if the total traversal time(cost) of these two different generated path is the same.

Table 5.2 and Fig 5.10 shows the result of our regular path planning solution and the solution using scalability optimization. The x-axis shows the amount of nodes in height/width of the generated graph diagram. The y-axis shows the computation time in milliseconds. In the result of two path generation model, the path generation time of the first local is noticeably smaller compared to the time of the second local map and the path generation time by only one main map. The reason is that the first model only needs to generate paths from only one departure to limited four ports communicated with the next local map. However, the second process needs to calculate all possible paths from the four ports departure to the destination and select the combined path with the lowest cost. Based on the generated result, we can notice that it's important to keep the map size to optimize the performance. In the map size of 100 nodes, the two path generation model can save around 50 percent of total path generation time. But if we increase the map size to 2500 nodes, the two path generation model can only 9 percent of the path generation time or even worse. The main reason that we can conclude is that the time complexity of the proposed method is $O\left(n^{2}\right)$. This liner map dividing method will perform better if we keep the local map size smaller (around 100 nodes).

If we divide the main map (size 20 by 10) into four parts, connected it to each other using four ports and performance the four separated path planning model separately. The performance of the divided map may be even worse compared with traversaling in 


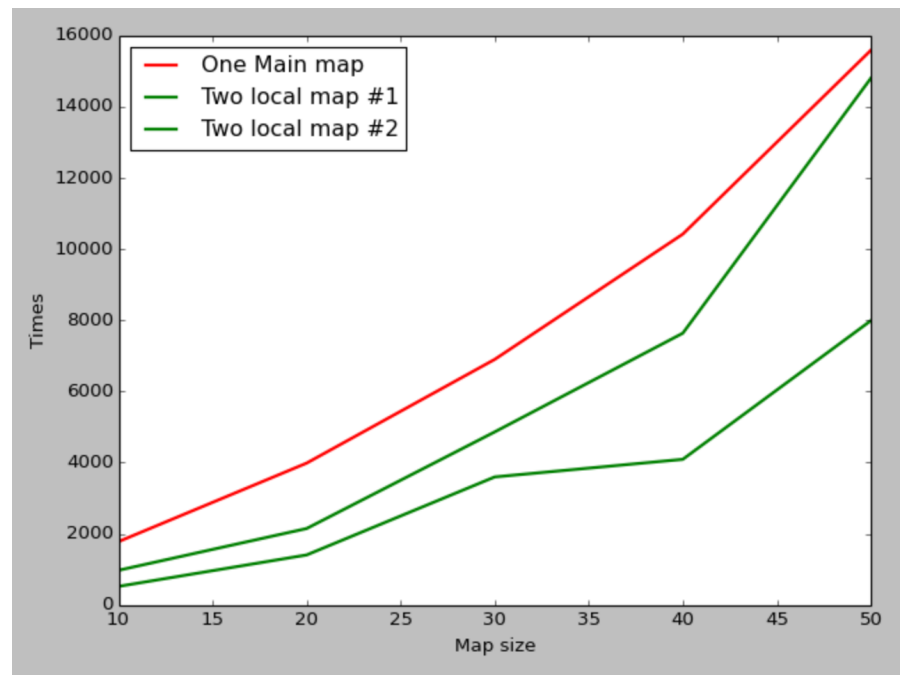

Figure 5.10. Path generation time by one main map (red line) and two local maps (green line).

Table 5.2. Path generating time by one main map and two local maps.

$\begin{array}{llllll}\text { Nodes } & 10 * 10 & 20 * 20 & 30 * 30 & 40 * 40 & 50 * 50 \\ \text { One Main map } & 1777 & 3984 & 6900 & 10422 & 15590 \\ \text { Two local map \#1 } & 518 & 1402 & 3595 & 4090 & 7989 \\ \text { Two local map \#2 } & 971 & 2145 & 4861 & 7636 & 14804\end{array}$

a single main map. This is because four ports for communication can result in calculating the optimal path in 16 times. The time complexity will be $O\left(k^{2} \frac{n^{2}}{v}\right)(\mathrm{k}$ is the number of ports, " $\mathrm{n}$ " is the number of nodes, and "v" is the number of divided map). Some parallel shortest path approach ${ }^{19}$ actually creates a cluster of vertices and connect them together using one edge, which is actually 'one port' connection of our map dividing method. Table 5.3 shows that if we only connect each local map using one port, four local maps solution will perform better. In the local map size of 10 by 10 , it can save 65 percent of the time. In this case, this time complexity of the proposed scalability solution will be $O\left(\frac{n^{2}}{v}\right)$ (" $\mathrm{n}$ " is the number of nodes and "v" is the number of divided map). 
Table 5.3. Path generating time by one main map and four local maps(max time).

$\begin{array}{llll}\text { Nodes } & 20 * 10 & 40 * 10 & 80 * 10 \\ \text { One Main map } & 3634 & 4779 & 7926 \\ \text { Four local maps } & 890 & 1675 & 2491\end{array}$




\section{Conclusions}

In connected vehicle technology, every car is connected and the necessary information is communicated to the surrounding cars. This technology promises that the transportation system can be enhanced in a more intelligent and efficient way. Using this Technology, our proposed method can provide more efficient navigation experience in the suburban city areas. Our approach considers real-time traffic taking into account blockages, V2V (vehicle to vehicle), V2I (vehicle to infrastructure).

Our methodology produces real-time optimal navigation routes in suburban traffic and we developed our method into a software system using Java tools. The method assists the driver by providing shortest routes, alternative paths based on map information and real-time data concerning connected cars. The method can handle an unexpected situation using a prediction algorithm based on car kinematic information. When there is a blockage, it predicts traffic delays due to blockage. Our method achieves real-time reaction in less than a second, predicts traffic blockage delays in about a second and updated routes in seconds. The traffic prediction goes beyond a simple reaction algorithm to an event by using lookahead anticipation, and predicting what other cars will do before the V2V confirmation. This provides even more advanced path planning prediction. The developed simulator take the Cleveland suburban area as an example. We 
conducted our proposed approach using this simulator to visualize the steps and results of path planning and traffic prediction.

Besides, we reduce the path generation time by dividing the main map into smaller local maps and conducting separated optimal path calculation. We reduce the path options by using preferred routes based on street properties (main/secondary street). This optimization can solve the problem of scalability issue of our proposed approach in large city metropolitan areas for generating optimal paths in a huge map. 


\section{Suggested Future Research}

\subsection{Path planning}

In our proposed method of path planning, we generate the optimal path in every path traversal from the departure to the destination. One possible improvement is creating the path from both ends (the current post and the destination) until these two paths reach to each other. This optimization can save the optimal path generation time.

In addition, the proposed path planning method is a non-direction search. Some tentative paths generated in the opposite direction from the destination may be redundant. One way to solve the problem is limiting the path to explore next by using the heuristic function like $\mathrm{A}^{*}$ algorithm ${ }^{20}$. In $\mathrm{A}^{*}$ pathfinding algorithm, the generated paths towards the opposite of direction will have a higher cost of heuristic function value, which represents the estimate of cost from the current node to the destination.

In our scalability solution, our proposed approach to the shortest path can still cost lots of computation resources since there may be lots of connected ports among local maps. One possible optimization is using historical data. In real life, although there are many road options, people can still prefer several traversal ways based on experience. 
This means many ports of local maps may be not worth consideration. Based on historical data, we can select the connected ports that we want to consider and save much resources.

\subsection{Traffic flow}

The current way of calculating the traversal cost of a street segment is using the notion of space mean speed. There are lots of different aspects that we can improve. In traffic prediction, non-parametric like recurrent neural network (RNN) may out-performance the parametric space mean speed formula proposed before. Some research exhibit that RNN with training based on time sequence dataset including traffic flow, occupancy, speed, weather, accident rates, etc, can forecast the real-time traffic situation 212223 . 


\section{Appendix A Simulator guide}

This simulator was implemented in Java using JDK 10, but it's compilable with any JDK versions that are newer than 8. It's built on the top of Java Swing and Java AWT. Running the main class will show a window with a pre-defined map powered by two input CSV files (node.csv and road.csv) which describe the node's location and road's connectivity, name, cost respectively. There is a blue button with an exclamatory mark on the bottom of the windows, which is the description of how to set destination, departure, edit the segment cost or even add new nodes. Once a destination and a departure is defined. We can click the green play button to show the optimal path based on this current traffic. We can also update the traffic to see if it will provide a different preferred path. Another way to edit the traffic of a particular street segment is offering the car speed and the length of the road of this street segment. For example, an input string value of "35,35,25,5" for the input dialog box means three cars in the segment with the current speed of 35, 35, $25 \mathrm{mph}$ separately and the length of the road is 5 miles. 


\section{Appendix B Scalability implementation guide}

The scalability implementation is also built on top of Java. The JDK version should be newer than 8. In the beginning, a graph diagram for simulating the map is constructed randomly based on pre-defined width and height. We used a queue for saving the search path and a map for saving the nodes regarding their lowest traversal cost. In this case, whenever we perform a new path generation, we need to clean the search 'cache' result saved in the map. In this implementation, we provided the time estimation of one, two and four path generation modes. One model directly traverses the whole graph from the up left corner to the down right corner. Two and four model divides the generated graph equally into two and four local map graphs, then traverse the graph separately and combined the minimum cost. Because the calculation of each local map is independent of each other, we processed the divided maps from top to bottom, passed the calculated result through four ports, and calculated their processing time separately. However, the processing time of each local map should be the same if we use some other multithreading techniques since they both have the same computing load and the same computing environment. All one, two and four models will provide a final path cost as the output along with the processing times. The output cost represents their estimated total traversal cost from the start to the end, and these three models should generate three same costs as a result. We verified the correctness of our program by checking if the three generated total traversal cost is the same.

The main class of scalability code

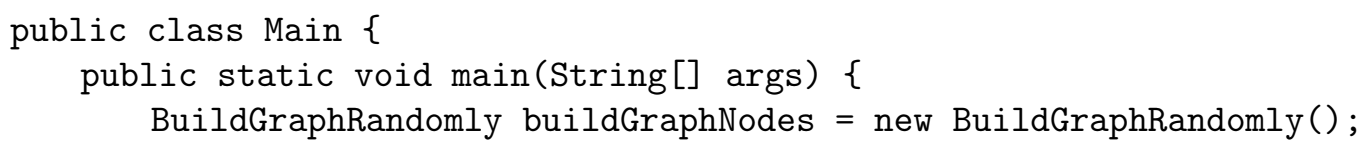




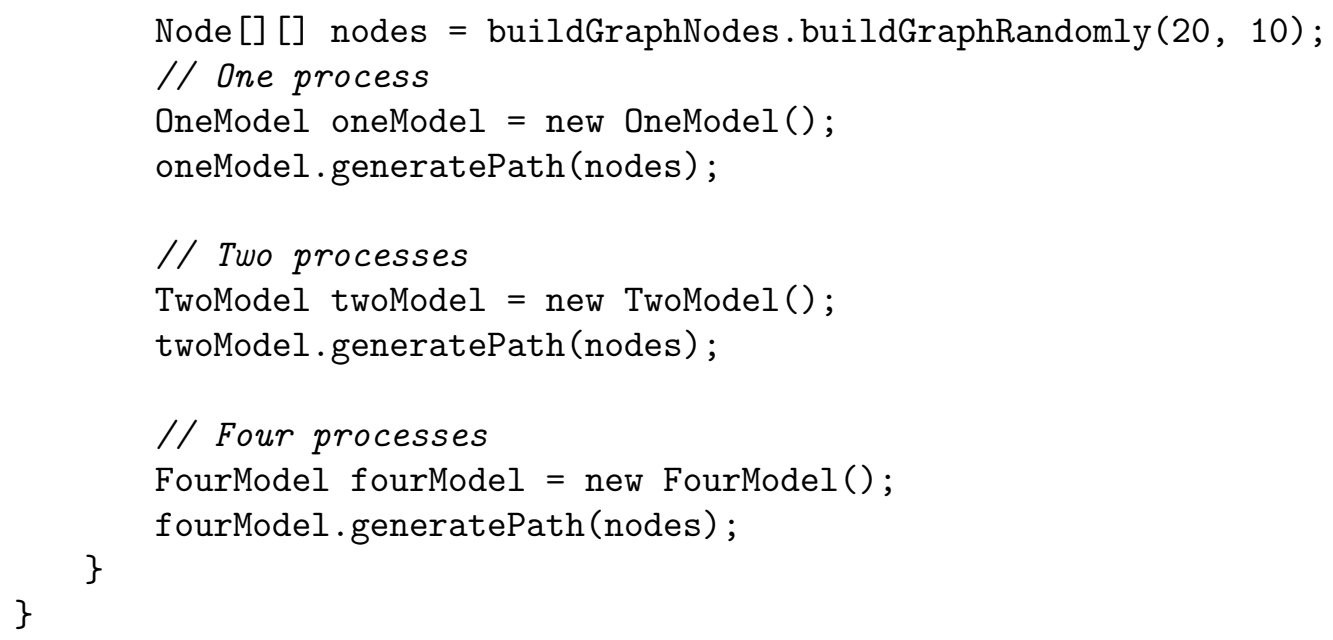

The class of node

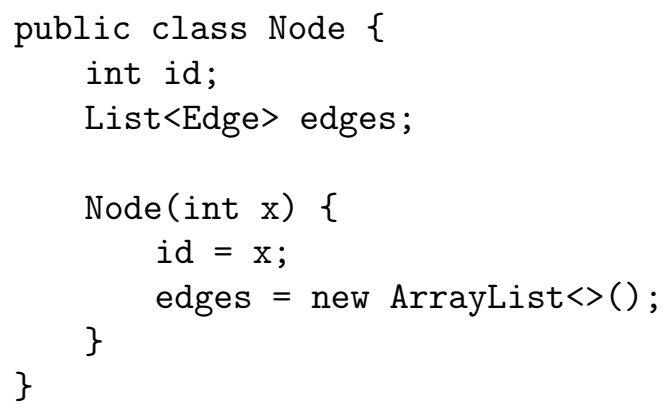

The class of edge

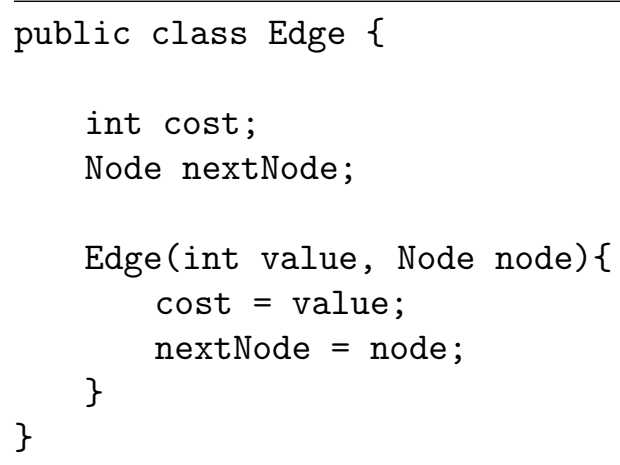

The class of building test graph

import java.util.Random; 


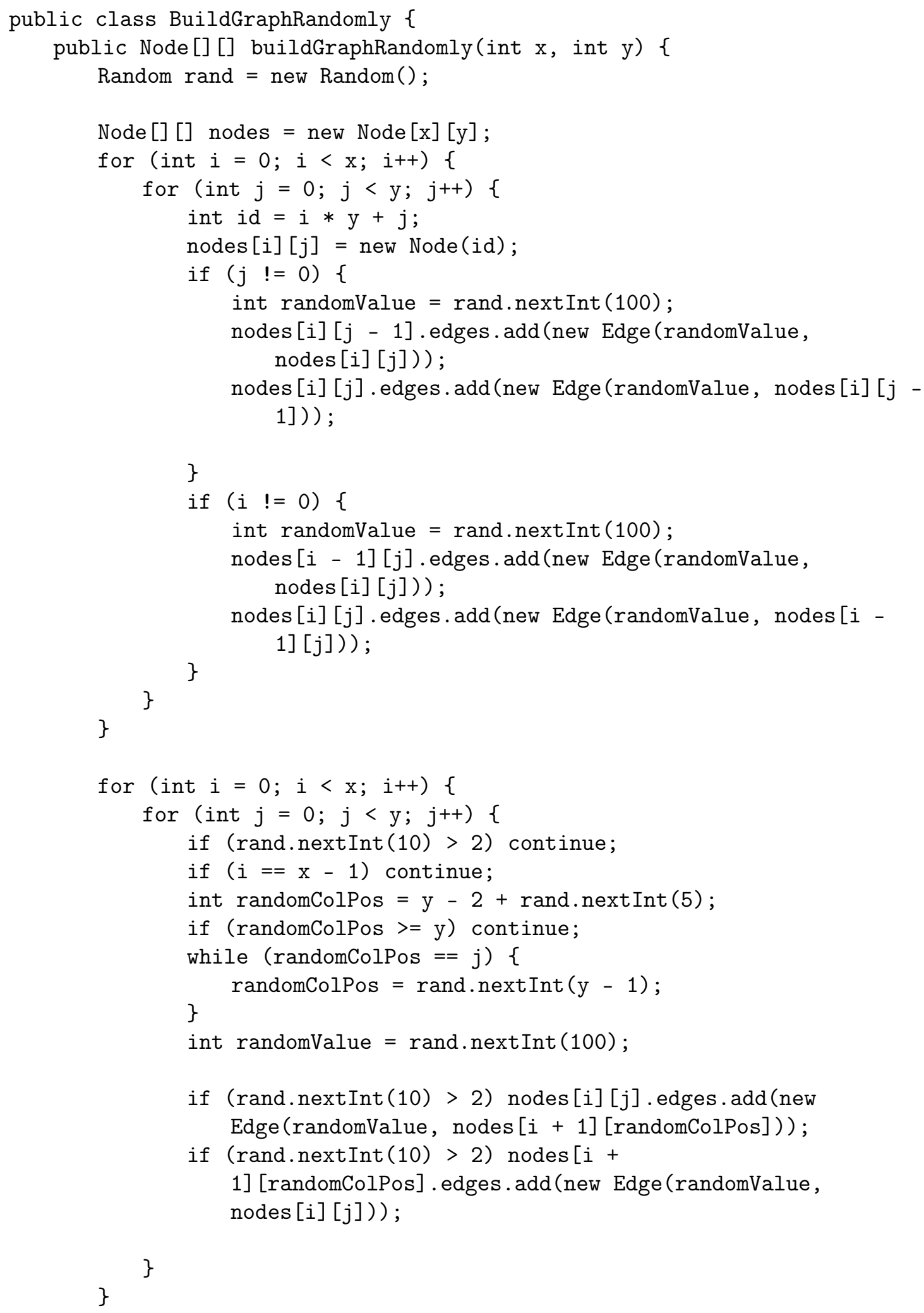




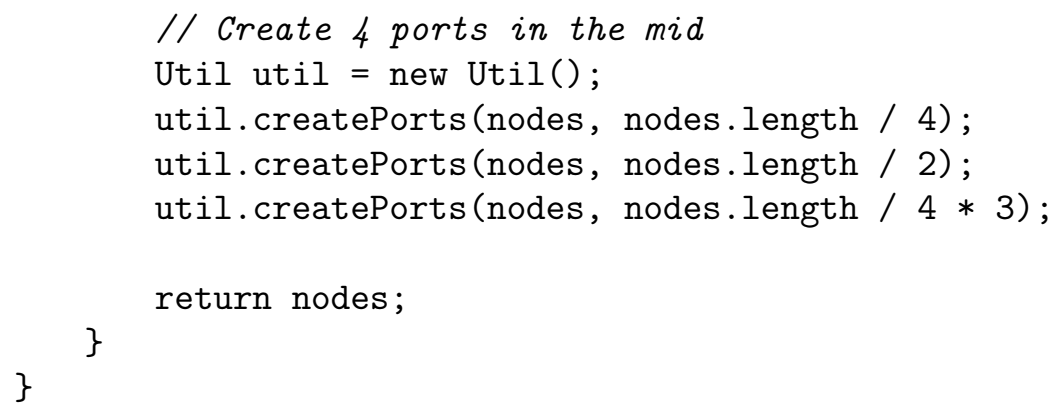

Calculating the cost of a divided local map

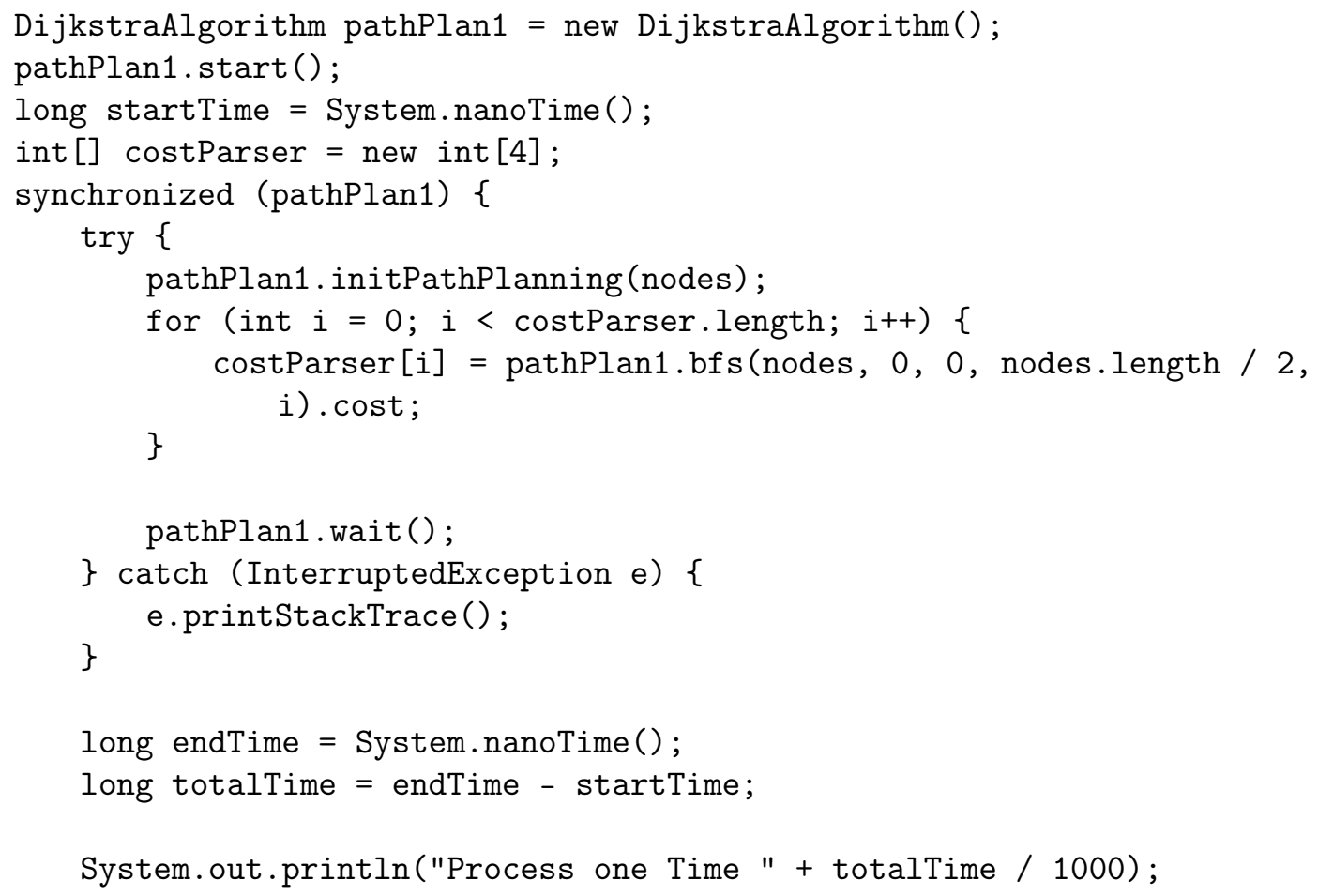

Path traversal

public Index bfs(Node[][] nodes, int startX, int startY, int endX, int endY) \{

Node startNode $=$ nodes [startX] [startY];

Node endNode $=$ nodes [endX] [endY];

Queue $<$ Node $>$ queue $=$ new LinkedList $<>()$;

queue.offer (startNode);

distMap.put (startNode, 0); 


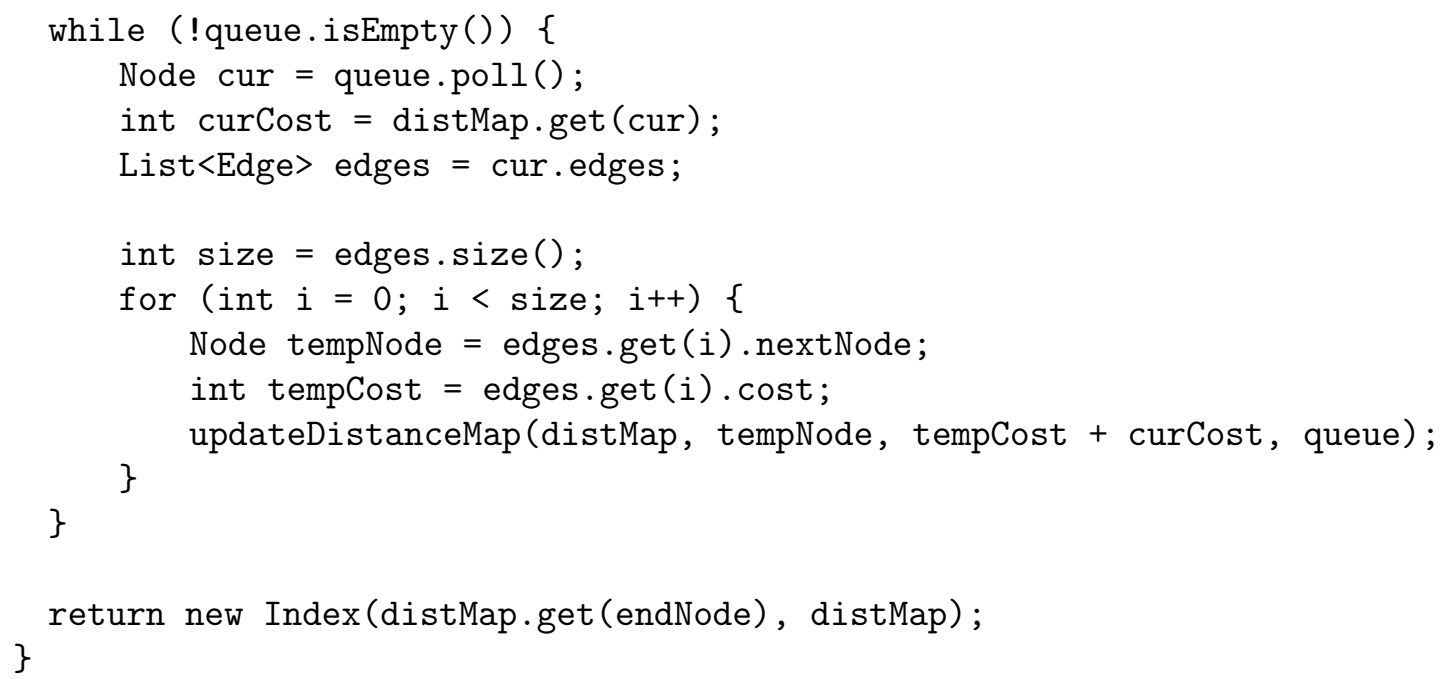




\section{Complete References}

[1] Kristen Hall-Geisler. All new cars could have v2v tech by 2023, Feb 2017.

[2] The U.S. Department of Transportation's (USDOT's) Connected Vehicle Program. Its research 2015-2019 connected vehicles.

[3] Get to know connected vehicle technology: V2v, v2x, v2i, Feb 2018.

[4] Antuan Goodwin. Audi's new traffic light information makes red lights less painful, 2016.

[5] Ning Lu, Nan Cheng, Ning Zhang, Xuemin Shen, and Jon W Mark. Connected vehicles: Solutions and challenges. IEEE internet of things journal, 1(4):289-299, 2014.

[6] Donald B. Johnson. A note on dijkstra's shortest path algorithm. J. ACM, 20(3):385388, July 1973.

[7] P. Bocci, J. Buchalo, S. Levenson, R. Noens, and S. Propp. Connected cars on wireless roads: Making it a reality. In IEEE Vehicular Technology Conference, pages 1-3, Sept 2006.

[8] J. Mosyagin. Using 4g wireless technology in the car. In 2010 12th International Conference on Transparent Optical Networks, pages 1-4, June 2010.

[9] X. Wu, S. Subramanian, R. Guha, R. G. White, J. Li, K. W. Lu, A. Bucceri, and T. Zhang. Vehicular communications using dsrc: Challenges, enhancements, and evolution. IEEE Journal on Selected Areas in Communications, 31(9):399-408, September 2013.

[10] Bob Williams. Intelligent Transport Systems Standards. Artech House, 2008.

[11] Conner Forrest. The x-factor in our driverless future: V2v and v2i, Feb 2018.

[12] Yacine Khaled, Manabu Tsukada, José Santa, and Thierry Ernst. The role of communication and network technologies in vehicular applications. CoRR, abs/1605.00889, 2016.

[13] Nicholas J. Garber and Lester A. Hoel. Traffic and Highway Engineering. Cengage Learning, 2014.

[14] Wikibooks. Fundamentals of transportation/traffic flow - wikibooks, the free textbook project, 2018. [Online; accessed 14-October-2018]. 
[15] L.C. Davis. Modifications of the optimal velocity traffic model to include delay due to driver reaction time. Physica A: Statistical Mechanics and its Applications, 319(Supplement C):557 - 567, 2003.

[16] Iamareebjamal. iamareebjamal/dijkstra-gui, Feb 2017.

[17] Wikipedia contributors. Swing (java) — Wikipedia, the free encyclopedia, 2018. [Online; accessed 9-November-2018].

[18] Wikipedia contributors. Abstract window toolkit - Wikipedia, the free encyclopedia, 2018. [Online; accessed 30-November-2018].

[19] Ye, Zilong. An implementation of parallelizing dijkstra's algorithm.

[20] P. E. Hart, N. J. Nilsson, and B. Raphael. A formal basis for the heuristic determination of minimum cost paths. IEEE Transactions on Systems Science and Cybernetics, 4(2):100-107, July 1968.

[21] D. Kang, Y. Lv, and Y. Chen. Short-term traffic flow prediction with lstm recurrent neural network. In 2017 IEEE 20th International Conference on Intelligent Transportation Systems (ITSC), pages 1-6, Oct 2017.

[22] T. Epelbaum, F. Gamboa, J.-M. Loubes, and J. Martin. Deep Learning applied to Road Traffic Speed forecasting. ArXiv e-prints, October 2017.

[23] Maher Ibrahim and Biswajeet Pradhan. Severity prediction of traffic accidents with recurrent neural networks. Applied Sciences, 7, 042017. 